\title{
eJRIEPS
}

Ejournal de la recherche sur l'intervention en éducation physique et sport

28 | 2013

Varia

\section{Styles de supervision de maîtres de stage en éducation physique : prise en compte du vécu émotionnel des stagiaires lors d'un entretien post- leçon}

Associate-teachers' styles of supervision in physical education: consideration of the emotional experience lived by pre-service teachers during a debriefing after lesson

\section{François Vandercleyen, Cécile Delens et Ghislain Carlier}

\section{OpenEdition}

Journals

Édition électronique

URL : http://journals.openedition.org/ejrieps/2899

DOI : 10.4000/ejrieps.2899

ISSN : 2105-0821

Éditeur

ELLIADD

\section{Référence électronique}

François Vandercleyen, Cécile Delens et Ghislain Carlier, «Styles de supervision de maîtres de stage en éducation physique : prise en compte du vécu émotionnel des stagiaires lors d'un entretien postleçon », eJRIEPS [En ligne], 28 | 2013, mis en ligne le 01 janvier 2013, consulté le 20 novembre 2019. URL : http://journals.openedition.org/ejrieps/2899 ; DOI : 10.4000/ejrieps.2899

La revue eJRIEPS est mise à disposition selon les termes de la Creative Commons Attribution 4.0 International License. 
Styles de supervision de maîtres de stage en éducation physique : prise en compte du vécu émotionnel des stagiaires lors d'un entretien post-leçon

François Vandercleyen, Cécile Delens et Ghislain Carlier

Université catholique de Louvain (UCL). Faculté des Sciences de la Motricité (FSM/EDPM). Groupe de Recherche sur la Socialisation, l'Education et la Formation (GIRSEF).

\section{Résumé}

Ancrée dans le contexte de l'intervention en éducation physique, la présente recherche vise à comprendre les pratiques d'accompagnement des maîtres du stage à travers leur prise en compte des émotions vécues par les stagiaires. A la lumière de la typologie de Brûlé (1983), l'objectif est de mettre en évidence les objets, les comportements et les styles de supervision de deux maîtres de stage au profil contrasté. Cette typologie distingue trois grands styles de supervision, non mutuellement exclusifs : directif, démocratique et expérientiel. A partir d'entretiens d'autoconfrontation croisés, l'analyse du discours post-leçon des maîtres de stage révèle certains invariants : un style directif, des comportements d'évaluation et d'instruction ainsi que les techniques de la communication comme objet principal de supervision. L'analyse dégage également le profil singulier de chacun d'eux, issu de la combinaison originale des trois styles. Les résultats montrent que le vécu émotionnel du stagiaire n'est pas abordé de manière frontale, mais plutôt de manière discrète ou détournée, en mettant l'accent sur d'autres objets de supervision. Finalement, cette étude souligne l'importance des émotions dans la formation des futurs enseignants, considérées comme source de développement professionnel.

Mots-clés : accompagnement, expérience de stage, incident critique, formation, entretien d'autoconfrontation

\section{Introduction}

La période de stage pratique en immersion dans un établissement scolaire, prévu dans la plupart des programmes de formation initiale à l'enseignement, représente pour de nombreux étudiants une étape cruciale de leur développement pré-professionnel (Altet \& 
al., 2002 ; Molina \& Gervais, 2008). Ce moment est souvent perçu par l'étudiant comme celui où il apprend vraiment (Boudreau, 2001 ; Serres et al., 2006).

Plusieurs recherches (Carlier, 2002, 2009 ; Leriche et al., 2010 ; Raymond \& Lenoir, 1998) montrent que le succès du stage dépend en majeure partie du type d'intervention du maître de stage auprès du stagiaire, ainsi que de la qualité de la relation entre les deux acteurs. Elles mettent notamment en évidence le double rôle présumé du maître de stage, qui doit être à la fois capable d'expliquer ses propres gestes pédagogiques au stagiaire, et dans le même temps, d'aider ce dernier à expliciter ses pensées, ses actions, ses décisions. Certaines insistent plus particulièrement sur l'importance de la prise en considération des émotions vécues par les stagiaires : « Le maître de stage doit être avant tout un soutien émotionnel indispensable pour des stagiaires régulièrement déstabilisés par leur confrontation avec la réalité du terrain» (Herbert \& Worthy, 2001, p. 910). D'autres, au contraire, mettent en garde contre le risque, pour les maîtres de stage, de se retrouver eux-mêmes « dans l'émotion », et que ce soutien émotionnel prenne le pas sur un véritable accompagnement réflexif (Chaliès \& Raymond, 2008).

De manière générale, les discours prônés par les instituts de formation voudraient que les maîtres de stage interviennent différemment selon l'étape de développement atteint par le stagiaire. Ainsi, la plupart des études consacrées à l'accompagnement des stagiaires insistent sur l'importance d'adapter le type de supervision pédagogique en fonction des besoins et des attentes des étudiants en formation (Boudreau, 2009 ; Boutet \& Rousseau, 2002 ; Carlier, 2002 ; Desbiens et al., 2009). De manière surprenante, peu d'études ont réellement investigué l'adéquation entre l'intervention du maître de stage et les besoins du stagiaire. Certaines ont étudié de près les différentes modalités de fonctionnement des maîtres de stage. D'autres ont appréhendé les attentes et les motivations des stagiaires. D'autres encore ont exploré les interactions entre les uns et les autres. Mais aucune - à notre connaissance - ne s'est intéressée à la façon dont le maître de stage tient compte du besoin d'expression des émotions du stagiaire.

Ancrée dans le domaine de la recherche sur l'intervention en éducation physique, la présente étude vise à comprendre comment les maîtres du stage s'y prennent pour prendre en compte le vécu personnel, et plus particulièrement la dimension émotionnelle du stagiaire. A quoi sont-ils attentifs lorsqu'ils accompagnent le stagiaire ? Quels sont leurs styles privilégiés de supervision après la séance ? A la lumière de la typologie de Brûlé (1983), l'objectif est de caractériser les pratiques d'accompagnement des maîtres de stage. 


\section{eJRIEPS 28 janvier 2013}

\section{Contexte théorique}

Après avoir défini les contours de l'accompagnement, nous présentons la typologie des styles de supervision de Brûlé (1983) comme référent théorique principal de la présente étude. Adaptée au contexte de l'éducation physique, cette typologie nous permet de mettre en évidence les objets, les comportements et le style des maîtres de stage.

2. 1. Une définition minimale de l'accompagnement

Très à la mode, la notion d'accompagnement se retrouve dans tous les domaines qui touchent, de près ou de loin, aux dispositifs et/ou aux pratiques de formation (Donnay et al., 2001 ; Paul, 2004). Cette notion n'est donc pas spécifique au domaine de l'éducation ni de l'enseignement. Bien au contraire, l'accompagnement est présent également dans le domaine du management des entreprises par exemple ou dans la sphère économique du travail social en général. Que ce soit dans le langage courant ou utilisé dans le monde professionnel, le concept d'accompagnement a été passablement abusé et semble avoir perdu de son sens et de ses valeurs initiales (Robo, 2009). Dépendamment du contexte, si le mot reste identique, il peut désigner des contenus parfois très différents. Compte tenu de cette polysémie, une définition précise de l'accompagnement, dans le contexte de la formation initiale des enseignants, s'impose.

Selon Paul (2004), le terme accompagnement doit être considéré comme générique. Or, “ on peut conseiller, orienter, aider, former, sans pour autant accompagner» (p. 78). L'accompagnement ne représente donc pas un concept à lui seul, mais suppose la convocation d'autres concepts qui le compose pour le définir. Conseil, tutorat, supervision, consultance, soutien, relation d'aide, parrainage, compagnonnage ou encore counselling, coaching, sponsoring, mentoring... Autant de pratiques, considérées comme des formes d'accompagnement (Dugal, 2008 ; Paul, 2004). Des concepts, parfois similaires, souvent complémentaires, qui s'apparentent à la notion d'accompagnement, sans la définir pour autant!

La supervision est souvent confondue avec l'accompagnement, notamment dans les pays anglophones. La notion de supervision appartient historiquement aux Etats-Unis, au domaine de la gestion. II s'agit du premier niveau du management, le niveau de direction en contact direct avec le travail de base (Bujold, 2002). Etymologiquement, superviser signifie « regarder au-dessus de » et correspond à «l'activité par laquelle la personne qui supervise guide, contrôle et révise le travail » (Le Petit Robert, 2007). La supervision est dite «pédagogique » si elle comporte une dimension de formation (Bujold, 2002). 


\section{eJRIEPS 28 janvier 2013}

Selon Donnay \& Charlier (2006), l'accompagnement est essentiellement une « affaire de médiation ». Exercer une médiation en pédagogie, “c'est favoriser l'établissement d'une relation de qualité entre un apprenant et un objet d'apprentissage » (Boutet \& Rousseau, 2002, p. 29). Cette activité de médiation met l'accent sur la prise en compte de l'expérience du sujet. Elle s'appuie sur trois principes : le retour sur l'expérience, la prise en compte des perceptions et la réévaluation de l'expérience. Une relation de médiation favorise ainsi l'émergence d'un savoir-enseigner, au service de la construction d'une identité professionnelle (Boutet \& Rousseau, 2002). II ne s'agit plus d'une relation « sur », mais d'une relation « avec » l'autre (Donnay \& Charlier, 2006).

Soutenues par une relation de médiation, toutes ces formes d'accompagnement se définissent comme des pratiques au sein desquelles un professionnel essaie d'être au plus près de la personne, dans l'exploration d'une situation problématique ou d'une épreuve à franchir. Leur dénominateur commun est de placer la relation au cœur du processus, dans la mesure où la fonction de l'un est de faciliter l'apprentissage de l'autre (Donnay, 2001 ; Donnay \& Charlier, 2006 ; Saint-Arnaud, 2003). D’après Paul (2009), le recours à une de ces formes d'accompagnement s'impose à un moment donné, compte tenu des exigences du milieu et des besoins individuels. "S'il arrive au moment opportun, même prescriptif, ce recours peut contribuer à opérer un tournant dans une vie et avoir des retombées au-delà de l'individu sur son environnement relationnel et social » (p. 101). Au-delà de ces pratiques qui composent l'accompagnement sans le définir complètement, il nous paraît important de revenir à l'origine étymologique du terme : « accompagner », c'est « faire compagnie avec », c'est-à-dire, « se joindre à un compagnon pour aller où il va en même temps que lui », le compagnon étant littéralement: «celui avec qui on partage le pain » (Le Petit Robert, 2007). Au sens large, l'accompagnement reviendrait à parcourir un bout de chemin ensemble, avec une action sous-jacente de soutien mutuel. Le sens étymologique du terme d'accompagnement permet de mettre en évidence la structure interne, constitutive de toutes les formes ou autres pratiques d'accompagnement nommées précédemment. Cette structure est constituée d'une double dimension fondamentale : la relation et le cheminement.

“ Accompagner l'autre, c'est être avec et aller vers, c'est-à-dire en même temps que lui, à son rythme, à sa mesure et à sa portée (Paul, 2009, p. 95). La personne ne peut être accompagnée que vers elle-même, vers le lieu de sa propre potentialité. II s'agit donc d'aider un individu à faire des choix, où la mise en relation (avec) est la condition de la 


\section{eJRIEPS 28 janvier 2013}

mise en chemin (vers). La dynamique de changement dépendrait donc de la qualité de la relation. L'idée d'un cheminement prévaudrait sur celle d'un but à atteindre.

Plusieurs auteurs (Donnay \& Charlier, 2006 ; Loizon, 2006 ; Boudreau, 2009 ; St-Arnaud, 2003) partagent cette définition, à la fois fondamentale et minimale, de l'accompagnement. Cependant, quatre points de tensions entre cette conception idéale de l'accompagnement et la réalité peuvent être relevés (Robo, 2009 ; Dugal, 2008) :

- être accompagné se fait au détriment d'une relation librement consentie ;

- atteindre des résultats (réussite du stage) se fait au dépend du choix des objectifs à réaliser par le stagiaire ;

- accompagner dans l'urgence (contrainte des deux parties) se fait au détriment du temps nécessaire à un accompagnement « en profondeur »;

- accompagner et en même temps évaluer : deux activités contradictoires.

Si la définition proposée par Paul (2009) a le mérite de pointer l'essence même de l'accompagnement en définissant sa structure interne, elle reste néanmoins une conception très idéaliste - voire idéalisée - , sans doute (très ?) éloignée de la pratique réelle des maîtres de stage. Cette façon de définir l'accompagnement ne nous est en effet que de peu d'utilité pour mettre en évidence les actions concrètes et qualifier le type de relation mis en œuvre par les maîtres de stage pour encadrer les stagiaires.

2. 2. La recherche sur les pratiques d'accompagnement des maîtres de stage D'après Leriche et al. (2010), les recherches sur les pratiques d'accompagnement mises en place par les maîtres de stage ont été réalisées selon trois orientations principales : 1) identifier et analyser la fréquence des pratiques d'accompagnement au cours du stage ; 2) analyser le contenu des entretiens post-leçons à partir de catégories prédéterminées ; 3) identifier le sens que les maîtres de stage et les stagiaires attribuent aux pratiques d'accompagnement. Notre recherche s'inscrit dans la deuxième orientation, avec l'originalité de s'appuyer sur l'observation des pratiques réelles des maîtres de stage tout en respectant l'écologie de la dyade (c'est-à-dire les interactions spontanées).

Par ailleurs, lorsqu'on analyse les pratiques d'accompagnement, il paraît judicieux de bien préciser de qui on parle exactement. En effet, chaque dispositif de formation détient ses spécificités, qui parfois rendent opaques ou incomparables certains résultats de recherche. Chaque pays y va de sa nomenclature, occasionnant de multiples confusions d'un contexte à l'autre. Dans notre contexte comme dans la plupart des dispositifs de formation, trois types d'intervenants peuvent être distingués : le(s) formateur(s), le(s) superviseur(s) et le(s) maître(s) de stage. Le premier type d'intervenant («formateurs ») 


\section{eJRIEPS 28 janvier 2013}

correspond aux enseignants mandatés par un institut pour intervenir dans la formation à l'enseignement des étudiants. Le second type («superviseurs ») fait référence à un représentant de l'institut de formation - souvent un enseignant - qui joue le rôle de médiateur entre le maître de stage et l'étudiant-stagiaire. Sous la forme d'une relation d'aide, il a pour mission d'aller, ponctuellement en stage, à la rencontre de la dyade, pour les soutenir, donner un avis et/ou évaluer. La troisième famille d'intervenants (« maîtres de stage ») désigne l'enseignant responsable d'accompagner l'étudiant-stagiaire dans son établissement scolaire qui, en lui mettant à disposition son groupe-classe pendant une durée déterminée, tente de l'aider à accomplir le passage vers la vie professionnelle. L'appellation « maître de stage » correspond à celle de «conseiller pédagogique » en France, « enseignant-associé » (cooperating teacher) ou « enseignant-accompagnateur » dans les pays anglo-saxons (Etats-Unis, Canada).

Dans le cadre de cette étude, il s'agit bien du rôle des «maîtres de stage » qui est questionné à travers l'analyse de leurs pratiques de supervision ou d'accompagnement (les deux termes étant utilisés dans ce texte de manière équivalente). Donnay et al. (2001) ont listé les différents rôles que peuvent endosser les maîtres de stage :

- rôle d'expert, de modèle d'enseignement et d'évaluateur ;

- rôle de conseiller, d'observateur, d'organisateur voire d'inducteur d'action ;

- rôle de collaborateur, de personne ressource et de soutien affectif ;

- rôle de consultant et d'intégrateur dans la profession, avec une fonction de socialisation et d'autonomisation ;

- rôle de compagnon, de guide, de médiateur et de praticien réflexif.

Pour approcher au plus près les pratiques réelles d'accompagnement des maîtres de stage, nous avons choisi de nous appuyer sur la typologie des styles de supervision de Brûlé (1983). Psychologue de formation, cet auteur propose une approche intégrée des différents modèles dominants en supervision, définie comme « toute relation d'aide en contexte professionnelle» (p.4). S'agissant d'une typologie transversale (non-spécifique au domaine de l'enseignement), elle ne s'intéresse qu'à la relation, sans porter aucunement sur la tâche. Une autre caractéristique de cette typologie est d'être opérationnelle et de constituer un véritable outil pratique. Les paragraphes suivants s'attachent à décrire cette typologie, à présenter les principaux avantages et limites, ainsi qu'à préciser comment nous l'avons transposée au contexte de notre étude. 


\section{eJRIEPS 28 janvier 2013}

2. 3. Typologie des « styles de supervision » (Brûlé, 1983)

Partant du constat que la plupart des approches insistent davantage sur les rôles du superviseur, plutôt que sur les techniques qu'il utilise et les compétences qu'il manifeste par des comportements précis, Brûlé (1983) a construit un outil visant à mesurer le style de supervision d'un intervenant en formation pratique, indépendamment de la discipline. S'appuyant sur une revue de la littérature exhaustive de la supervision, il a mis au point une typologie qu'il a ensuite testée et validée statistiquement. De cette analyse statistique, il ressort deux grands styles indépendants (voir figure 1) : le style « didactique » (ou directif) et le style « expérientiel » (ou non-directif). Le style « démocratique » constitue une structure intermédiaire. Les deux premiers rassemblent deux conceptions a priori opposées de la supervision: l'une perçoit le maître de stage comme un expert de la discipline (plutôt « directif ») ; l'autre comme un consultant ou un thérapeute (plutôt « nondirectif »). La troisième catégorie, qualifiée d'intermédiaire, comprend un modèle intégrant les deux premières en attribuant un rôle de collègue ou de conseiller au superviseur. L'expert se préoccupe surtout des connaissances et de l'expérience qu'il veut transmettre au stagiaire; le collègue/conseiller accorde la priorité aux besoins personnels du supervisé ; tandis que le consultant/thérapeute se centre plutôt sur la relation avec le stagiaire, encourageant ce dernier à s'ouvrir à sa propre expérience.

Chaque style est défini par un certain nombre de comportements observables (cf. tableau 1). A la fois différents et complémentaires, les trois styles ne sont pas exclusifs mais peuvent tout à fait coexister. La combinaison originale et particulière des trois styles de supervision permet de dégager un profil d'accompagnement spécifique et particulier pour chaque maître de stage.

\begin{tabular}{|l|l|l|l|}
\hline \multirow{2}{*}{ Figure I. Typologie des « « styles de supervision » (Brûlé, 1983) } \\
\hline Styles & Directif & Démocratique & Expérientiel \\
\hline \multirow{2}{*}{ Comportements } & - Direction & - Clarification & - Consultation \\
& - Instruction & - Discussion & - Expression de \\
& - Évaluation & - Confrontation & soi \\
& - Sécurisation & - Démonstration & - Exploration \\
& - Provocation & & - Soutien \\
& & & - Permissivité \\
\hline \multirow{2}{*}{ Rôles } & Professeur/expert & Collègue/conseiller & Consultant \\
\hline
\end{tabular}




\section{eJRIEPS 28 janvier 2013}

\section{3. 1. Le style directif}

Le maître de stage qui adopte un style « directif » se considère plutôt comme un expert sur le plan des connaissances théoriques, adopte une attitude de leadership et exerce une certaine autorité. Dans une situation où il est celui qui maîtrise la meilleure forme d'intervention, le maître de stage aide le stagiaire à comprendre et à saisir rationnellement l'intervention pratique. II vise à conduire le stagiaire vers des normes objectives et stimule davantage l'acquisition de techniques spécifiques que d'attitudes ou de valeurs. II enseigne au stagiaire comment répondre à une situation problématique, en fonction de sa propre expérience. L'enseignant adoptant le style directif cherche donc à modeler les comportements du stagiaire en calquant sur ses propres comportements d'intervention.

\section{3. 2. Le style expérientiel}

Dans le style expérientiel, le maître de stage encourage le stagiaire à vivre sa propre expérience. Son but est de faire expérimenter divers concepts au stagiaire. II délaisse les éléments objectifs et purement cognitifs pour s'intéresser davantage à la sphère subjective du stagiaire, en approchant les sentiments et les émotions ressenties par ce dernier, en le suivant où il veut aller et en l'aidant à puiser dans ses propres ressources. Le maître de stage ne tient compte des éléments objectifs que si le stagiaire l'y conduit, sans le précéder, ni le diriger. II vise à faciliter l'exploration de ses propres actions et réactions et favorise l'initiative personnelle. Ce style permet au stagiaire de manifester les sentiments d'insécurité et de doutes que peut éprouver tout débutant. L'enseignant vise principalement ici à développer la sensibilité du stagiaire dans sa pratique, sa capacité à communiquer et à comprendre. Cinq comportements de supervision sont associés à ce style : la consultation, l'expression de soi, l'exploration, le soutien et la permissivité.

\section{3. 3. Le style démocratique}

Dans ce type de relation intermédiaire, le maître de stage se considère plutôt comme un ami ou un collègue du stagiaire, avec qui il souhaite entretenir des échanges d'opinion, sous la forme d'un dialogue ouvert, et au sein duquel l'un et l'autre sont disposés à s'entraider. Le maître de stage « démocratique » aide surtout le stagiaire à clarifier sa pensée, et se penche particulièrement sur les préoccupations problématiques de celui-ci. Il encourage et fait en sorte que le stagiaire trouve lui-même la solution à son problème. $A$ ce style se rapportent quatre comportements de supervision : la discussion, la clarification, la confrontation et la démonstration. 


\section{eJRIEPS 28 janvier 2013}

2. 4. Transposition de la typologie de Brûlé à notre contexte : intérêts et limites Du point de vue de la recherche, cette typologie des styles de supervision présente trois avantages majeurs (Vandercleyen, 2010) :

- Cette approche intègre différents modèles théoriques. Elle met l'accent non seulement sur les différences entre ces modèles, mais aussi sur leur complémentarité. Elle tend, non pas à mettre en valeur une approche en particulier, mais à donner une synthèse des différentes conceptions théoriques de la supervision accompagnante, issues d'autres modèles existants. Elle permet d'éviter la tendance à vouloir faire ressortir une approche idéale de la supervision, qui serait « meilleure qu'une autre ».

- Le second attrait du modèle de Brûlé est son caractère opérationnel. II s'agit d'un instrument concret, sous la forme d'un questionnaire, composé de nombreux items illustratifs de chacun des 14 comportements de supervision.

- Cet outil se révèle également fiable, dans la mesure où, expérimenté statistiquement, il démontre des qualités psychométriques satisfaisantes.

Du point de vue de la formation, le principal intérêt de cet instrument est d'aider les acteurs - tant les maîtres de stage que les stagiaires - à clarifier leurs difficultés et à autoévaluer leur mode de fonctionnement relationnel en cours de stage. Pour le maître de stage, il permet de prendre conscience de son propre style. II peut aussi lui permettre de dénouer une situation conflictuelle, en clarifiant les attentes des uns et des autres (Brûlé, 1983). Le stagiaire, quant à lui, peut se servir de cet outil pour comprendre la façon de se sortir d'une impasse ou d'une relation difficile vécue avec son maître de stage, mais aussi de confronter ses perceptions avec ce dernier.

En revanche, cet outil présente également certaines limites. La non-spécificité de l'outil par rapport à une discipline en particulier constitue à la fois un avantage et une faiblesse. L'avantage, souhaité par l'auteur, est d'être un outil transversal, à savoir qu'il peut être utilisé dans différents contextes de supervision de stage. Du domaine de l'économie à la psychologie, en passant par l'éducation ou l'enseignement, cet outil reste valable à partir du moment où il s'intéresse exclusivement à la relation. Cet avantage se transforme assez rapidement en limite, dans la mesure où il suppose du coup quelques adaptations au contexte de recherche. Dans le cadre de cette étude, nous considérons les 14 échelles comme des catégories permettant une analyse de contenu (cf. méthodologie).

2. 5. Profil d'accompagnement des maîtres de stage L'objectif de la présente recherche est de décrire et de comprendre les pratiques d'accompagnement des maîtres de stage. La question centrale est la suivante : comment 


\section{eJRIEPS 28 janvier 2013}

le maître de stage s'y prend-il pour accompagner le « vécu émotionnel » du stagiaire lors d'un débriefing d'après séance?

Le « vécu émotionnel » peut être compris au sens de Ria et al. (2003), c'est-à-dire à la façon dont le stagiaire éprouve dans l'action un certain nombre de phénomènes subjectifs souvent éphémères ou fluctuants. Cette notion renvoie donc à l'expérience du stagiaire (« faire l'expérience de »), et plus précisément à l'expression de son propre ressenti situé dans un contexte précis (situated emotions). C'est l'idée que vivre une émotion, quelle qu'elle soit, se traduit par la sensation de baigner dans un univers particulier, diffus, coloré par cette émotion. Le vécu émotionnel est donc considéré ici selon une approche phénoménologique.

S'appuyant sur la typologie de Brûlé (1983), deux sous-questions constituent les principaux centres d'intérêts de cette étude :

- Quelle est la nature des observations réalisées par le maître de stage pendant la séance ? Comment récupère-t-il ces observations dans son discours d'après séance?

- Quel(s) est/sont le(s) style(s), les comportements, les objets de supervision du maître de stage qui témoignent de la prise en compte du « vécu émotionnel » du stagiaire dans son discours d'après séance?

A partir de différents travaux de synthèse (Boutet \& Rousseau, 2002 ; Chaliès \& Durand, 2001 ; Molina \& Gervais, 2008 ; Raymond \& Lenoir, 1998), l'hypothèse générale est que les maîtres de stage ont tendance à présenter un profil d'accompagnement plutôt directif, s'intéressant peu au ressenti du stagiaire. Par ailleurs, ils seraient davantage centrés sur la gestion de classe que sur la gestion des contenus disciplinaires (Leriche et al., 2010).

\section{Méthodologie}

Etant donnée la visée compréhensive de notre étude, nous avons opté pour une méthodologie de nature qualitative. Nous soutenons en effet une démarche heuristique, située plutôt du côté de la découverte que de la preuve. Si nous voulons comprendre la logique interne du maître de stage, il convient de prendre en compte son point de vue, dans toute sa dimension, avec toute sa complexité (Albarello et al., 2007). Ainsi, l'analyse qualitative par étude de cas présente l'avantage de permettre une description fine et riche des processus ancrés dans une situation spécifique, complexe et en pleine évolution (Van der Maren, 1995 ; Yin, 1998). 


\section{eJRIEPS 28 janvier 2013}

\section{1. Participants}

La recherche rend compte de deux études de cas contrastées : MSJ (Fabienne, maître de stage de Jean et MSM (Maxime, maître de stage de Marie).

Fabienne (MSJ) et le stagiaire Jean

Fabienne est une maître de stage âgée de 50 ans, qui dispose d'une expérience d'une trentaine d'année en accompagnement. Très impliquée dans son école et motivée par son travail, elle pratique le théâtre en dehors. Elle est donc très sensible aux techniques d'expression, à la comédie et à l'humour. A ce propos, elle dit aimer «taquiner les stagiaires et rire, non pas d'eux, mais avec eux ». Un de ses loisirs favori est d'ailleurs le cirque. Jean quant à lui est un stagiaire âgé de 22 ans qui n'a qu'une vingtaine d'heures de stage derrière lui. II s'agit de la quatrième heure de cours qu'il dispense avec cette classe, et donc avec cette MS. Lui-même ex gymnaste de haut niveau, il dispense une séance axée sur l'apprentissage de «l'appui tendu renversé » (ATR). La classe, mixte (filles-garçons), est composée de 26 élèves âgés entre 14 et 15 ans qui ont choisi l'éducation physique dans leurs cours « à option ».

Maxime (MSM) et la stagiaire Marie

Agé de 41 ans, Maxime est un maître de stage « novice » en la matière dans la mesure où il exerce la fonction de maître de stage pour la première fois. Marie constitue donc sa première expérience d'accompagnement. Enseignant depuis une bonne quinzaine d'années dans le même établissement scolaire, il exerce bénévolement au sein de son école la fonction de «médiateur» entre le corps professoral et les élèves jugés « difficiles » (manifestant des comportements d'indiscipline). Marie, stagiaire âgée de 21 ans, dispense seulement sa $12^{\mathrm{e}}$ heure de cours, la deuxième avec ce groupe-classe composé de 24 élèves (âgés entre 15 et 16 ans). Sa séance a pour objectif l'apprentissage de la manchette en volley-ball. Elle se dit non spécialiste de la discipline, préférant nettement la motricité expressive (danse, fitness...).

Ceux deux stagiaires s'inscrivent dans la formation initiale (de type universitaire) en didactique de l'éducation physique dispensée à la Faculté des Sciences de la Motricité (UCL). Les deux maîtres de stage quant à eux sont des enseignants en activité dans un établissement scolaire issu de la Fédération Wallonie-Bruxelles (Belgique). Ils sont reconnus pour leur expertise par leurs pairs, et travaillent en étroite collaboration avec l'institut de formation initiale (UCL). En endossant la responsabilité d'accompagner un stagiaire, ces maîtres de stage acceptent également d'adhérer à la philosophie de l'institut. La pédagogie des motivations représente l'ensemble des valeurs fondatrices de 


\section{eJRIEPS 28 janvier 2013}

la formation, laquelle peut se résumer par l'expression suivante : apprendre en s'amusant. Le plaisir d'avoir appris, mais également le plaisir immédiat ressenti lors de la pratique des APS à l'école, constitue le credo de la formation initiale à l'UCL.

Le choix de chacune des dyades s'est opéré sur la base de caractéristiques très contrastées de chacun des acteurs. Les stagiaires présentent à la fois des backgrounds différents, mais également un vécu émotionnel singulier. En effet, Jean ressent des émotions négatives intenses au cours de sa séance (anxiété majeure), tandis que Marie éprouve des sentiments négatifs plutôt diffus voire du plaisir à certains moments de sa séance (Vandercleyen et al., 2010). Les maîtres de stage ont également chacun une expérience particulière en termes d'accompagnement et une approche a priori différente : l'une est comédienne, avec une formation en art théâtral ; l'autre est médiateur, avec une formation en psychologique.

\section{2. Description du protocole}

Le protocole a été construit en deux étapes principales, l'une dite « interactive » et l'autre dite «post-active »: 1) l'observation filmée du stagiaire en séance, ainsi que le recueil simultané des commentaires «en direct » du maître de stage ; 2) le débriefing postséance, sous la forme d'un entretien d'autoconfrontation croisée avec le maître de stage et son stagiaire.

3. 2. 1. Observation filmée du stagiaire en séance et recueil simultané des commentaires du maître du stage

Le stagiaire est filmé durant son intervention en situation « naturelle » d'enseignement. Le maître de stage est également inclus dans le dispositif. Installé aux côtés du chercheur, à proximité de la caméra, il a pour mission de commenter «à voix haute » tout ce qu'il observe à propos des actions menées par le stagiaire. Cette implication, innovante, s'est d'abord imposée comme nécessaire pour respecter l'écologie du système " maître de stage - stagiaire ». II nous est apparu également pertinent dans le cadre de notre recherche d' "avoir accès » aux observations du maître de stage au sujet des actions menées par le stagiaire. L'intérêt de cette prise de donnée est double. D'une part, elle permet de confronter les indices perçus par le maître de stage avec ceux décelés par le stagiaire, et d'en apprécier l'éventuelle divergence ainsi que le décalage temporel potentiel. D'autre part, ce type de données particulier permet de corroborer l'avis du chercheur sur le choix des incidents critiques à analyser lors de l'entretien post-séance. Afin de réaliser ces prises de données, un dispositif technique particulier a été mis au point. II permet, non seulement d'enregistrer l'expression verbale et non verbale du 


\section{eJRIEPS 28 janvier 2013}

stagiaire, mais également de recueillir simultanément, en cours de séance, les commentaires «en direct » du maître de stage. La mise en œuvre de ce protocole unique relève d'un challenge technique : enregistrer les commentaires des deux acteurs sur la même bande image-son. Équipés tous deux d'un micro-cravate et d'un émetteur, les discours du maître de stage et du stagiaire ont été directement enregistrés, par l'intermédiaire d'une table de mixage, sur un ordinateur portable. En cours de séance, le chercheur est amené à exercer une attention partagée entre deux actions simultanées : observer et écouter. D'une part, il repère tout incident critique, illustrant une séquence susceptible d'être discutée par la suite. D'autre part, le chercheur écoute activement les commentaires du maître de stage, en vue de soutenir le discours spontané de celui-ci, tout en évitant de réagir verbalement. Si les silences se prolongent, les consignes sont rappelées sous la forme de relances comme : " n'hésite pas à me dire ce que tu observes en ce moment ! ». A la fin de la séance du stagiaire, il est demandé aux participants de s'abstenir de tout échange «à chaud». Le but de cette consigne est de réserver l'exclusivité des réactions des acteurs lors de l'entretien post-séance. Ce dernier remplace alors l'habituel débriefing spontané de fin de séance.

\section{2. 2. Entretien post-leçon selon la technique de l'autoconfrontation}

L'entretien post-séance prend appui sur le visionnement de séquences vidéo, lesquelles ont été sélectionnées préalablement. Concrètement, avant le début de l'entretien postséance, le chercheur visionne le film de la séance et identifie les incidents critiques relatifs à la gestion de classe. Par incident critique, il faut entendre « tout évènement inattendu repéré par un observateur externe qui témoigne d'un fait saillant dans une situation, où les intentions de l'action sont claires et les conséquences de l'action évidentes (Flanagan, 1954). Le choix des incidents est co-déterminé, par les observations du chercheur ainsi que par celles du maître de stage. Bien que le stagiaire ne participe pas directement au choix des incidents discutés, son point de vue est néanmoins pris en compte, puisqu'en début d'entretien, le stagiaire est invité à estimer le caractère « critique » de la séquence choisie.

La technique d'entretien par autoconfrontation (Theureau, 1992) vise à faire expliciter les pensées, les intentions, les émotions et le ressenti du sujet par une confrontation à ses propres actions, par l'intermédiaire d'un support filmé. L'entretien post-séance s'inspire de cette technique, choisie parmi d'autres car elle présente l'avantage de produire des verbalisations contextualisées, en connexion avec la situation réellement vécue. Après avoir revu la séquence vidéo illustrant l'incident critique, le stagiaire est invité à exprimer 


\section{eJRIEPS 28 janvier 2013}

son ressenti au sujet de l'incident. Toutes les séquences se sont avérées constituer « un problème ou une difficulté ». Cette corroboration a posteriori du stagiaire, concernant le choix des incidents par le chercheur, a permis de valider la pertinence des incidents sélectionnés. Le stagiaire est ensuite invité à verbaliser ses actions, ses intentions, ses sensations ainsi que ses émotions.

\section{3. Analyse de contenu}

Le verbatim issu de la retranscription du discours des maîtres de stage lors des deux prises de données (phases interactive et post-active) a été traité par analyse de contenu de type catégoriel. La procédure permettant cette analyse est décrite en deux points : 1) le découpage du verbatim en unités de sens ; 2) le processus de catégorisation. A noter que le codage des unités de sens dans chacune des catégories a été facilité par l'utilisation du logiciel informatique $\mathrm{N}-\mathrm{Vivo}^{\circledR}$.

3. 3. 1. Découpage du verbatim en unités de sens

Les verbatim sont découpés en « unités de sens » (Mucchielli, 1988). Il s'agit des énoncés possédant un sens complet en eux-mêmes, peu importe le nombre de caractères. Concrètement, les thèmes abordés par le maître de stage sont repérés. S'il passe d'une idée à une autre, le paragraphe est coupé en allant à la ligne, créant ainsi une nouvelle unité de sens.

\section{3 .2. Processus de catégorisation, adapté de Brûlé (1983)}

Une première catégorisation distingue les trois « styles de supervision » définis par Brûlé (1983), ainsi que chaque «comportement » s'y rapportant. L'ensemble des catégories décrites par Brûlé dans sa typologie a été reprise, à l'exception de trois comportements relatifs au style « expérientiel » : l'exploration, l'expression de soi et la consultation. Si le caractère discriminant de ces comportements a été prouvé, leurs définitions n'en restent pas moins très proches l'une de l'autre. Tous sont issus du style « expérientiel », leur point commun est de s'intéresser au ressenti vécu par le stagiaire : la consultation en tient compte, l'exploration stimule le stagiaire à se pencher sur son vécu « intérieur », et l'expression de soi l'aide à le verbaliser. C'est pourquoi, il est apparu judicieux de « condenser» ces trois comportements en un seul. Cette option théorique a notamment pour effet méthodologique de faciliter le codage de cette forme particulière de relation d'aide, centrale dans notre recherche.

Par ailleurs, un premier codage préliminaire nous a incités à créer une catégorie supplémentaire. En relisant les unités de sens contenues dans la catégorie « instruction », il nous est apparu pertinent de nuancer le comportement d'instruction par celui de conseil. 


\section{eJRIEPS 28 janvier 2013}

L'échelle d'instruction décrit le comportement d'un superviseur qui « donne des indications précises sur divers aspects théoriques et pratiques du stage, qui répond aux questions et lui indique comment interpréter ou améliorer une habileté » (Brûlé, 1983, p. 47). Nous avons défini le comportement de conseil lorsque le maître de stage « fait des propositions ou des suggestions au stagiaire sur certaines façons de procéder, voire apporte des solutions lui permettant de sortir d'une impasse, sans imposer pour autant son avis ». Dans le premier cas (instruction), le maître de stage se substitue au stagiaire pour résoudre le problème à sa place ; c'est pourquoi il s'agit bien d'un comportement directif ; alors que dans le second cas (conseil), l'enseignant fait une proposition au stagiaire, sans imposer sa manière de faire mais au contraire en lui laissant le choix ; c'est pourquoi ce comportement de conseil appartient, selon nous, au style « démocratique ». L'annexe 1 reprend les 13 sous-catégories retenues pour représenter l'ensemble des comportements de supervision des maîtres de stage, chacune étant définie et adaptée au contexte de la recherche.

\section{3 .3. Création d'une catégorie " objets de formation »}

Un deuxième type de catégorisation identifie les « objets de supervision » selon la nature des thèmes abordés par le maître de stage. Concrètement, la question suivante est posée : « sur quel aspect de la formation le maître de stage porte-t-il son regard lorsqu'il parle ? ». Cette seconde catégorisation s'est imprégnée du principe du modèle semiouvert, c'est-à-dire s'appuyant d'une part sur les catégories pré-existantes et, d'autre part sur des catégories « émergentes ». Les catégories pré-existantes sont construites à partir de la grille d'observation délivrée aux maîtres de stage par l'institut de formation initiale. Cette grille, intitulée « bilan de fin d'unité », rassemble une vingtaine d'indicateurs aidant le maître de stage à apprécier les compétences atteintes ou non par le stagiaire. D'autres catégories, dites émergentes, sont induites à la lecture des unités de sens. Au total, dix sous-catégories relatives aux « objets de formation » (cf. annexe 2) sont construites, à savoir : les objectifs (obj), la préparation (prepa), les contenus (cont), les techniques de communication (tcom), l'organisation (org), l'ordre et la discipline en classe (ordr), la relation entretenue par le stagiaire avec les élèves (rel), l'éthique (eth), la réflexivité (reflex) et le vécu personnel du stagiaire (vecu).

\section{3. 4. Description quantitative des résultats en « volume»}

Afin d'avoir une vue d'ensemble des données, un traitement quantitatif des données « en volume » est choisi. II s'agit d'une analyse sur la base de la répartition du nombre de caractères compris par catégories. Ce type d'analyse a l'avantage de présenter les 


\section{eJRIEPS 28 janvier 2013}

données de manière graphique et constitue une porte d'entrée intéressante à l'analyse et à l'interprétation des données. L'hypothèse est fondée sur le fait que, plus le volume est élevé, plus le maître de stage accorde de l'importance à cette catégorie. Afin de s'assurer de la validité de ce type d'analyse, une corrélation simple $(R)$ a été calculée entre les deux modes de traitement (par «nombre de caractères» et par « unités de sens »). Les résultats de ce calcul montrent des coefficients de corrélations élevés (entre 0.866 et 0.988 ) entre le volume en termes de caractères et le volume en termes d'unités de sens.

\section{3. 5. Critères de qualités de l'analyse}

Différents codages intra-juges ont été réalisés (Albarello et al., 2007), nécessitant plusieurs redéfinition des catégories entre chaque codage, jusqu'à parvenir à un score satisfaisant de $86 \%$ d'accord. Nous avons ensuite procédé à un codage inter-juge, réalisé en «double aveugle ». Après de nouveaux ajustements catégoriels, ce second codage s'est avéré concluant avec un coefficient d'accord de $84 \%$.

\section{Résultats}

Pour les deux études de cas, les résultats de l'analyse sont présentés en respectant une systématique en cinq temps, en lien avec les questions de recherche : 1) mise en évidence des objets d'observation du maître de stage pendant la séance ; 2) description du style, des comportements et des objets de supervision après la séance ; 3) mise en lumière d'une dynamique de supervision récurrente dans le discours post-séance. II s'agit de déceler si l'enseignant utilise des comportements de supervision selon un ordre spécifique, en fonction des objets de formation abordés ; 4) la mise en relation des discours du maître de stage pendant et après la séance ; 5) la détermination d'un profil spécifique pour chaque maître de stage.

L'analyse montre que chaque maître de stage interrogé présente un profil de supervision singulier, composé d'une combinaison particulière des trois styles de supervision mis en évidence par Brûlé (1983). Les principales singularités de chaque cas « maître de stage » étudié sont d'abord présentées, tandis que les invariants sont pointés dans la discussion.

4. 1. Fabienne, une maître de stage à la fois stimulante et sécurisante Concernant le contexte de cette première étude de cas, il est important de signaler que Fabienne et Jean ne se connaissaient pas avant le début du stage. Elle a proposé au stagiaire d'inscrire son cours dans un cycle de gymnastique centré sur l'apprentissage de l'ATR. II s'agit de la quatrième séance sur ce thème ; dernière séance avant l'évaluation des élèves sur cette compétence. S'appuyant sur une pratique personnelle de 


\section{eJRIEPS 28 janvier 2013}

gymnastique en club, Jean dit se sentir à l'aise dans l'enseignement de cette discipline. II estime être très compétent dans la maîtrise des contenus. En revanche, une discussion préalable avec Fabienne laisse entendre que Jean est en difficulté majeure sur le plan de la gestion de classe, alors même que le groupe-classe est plutôt discipliné. Cet avis est corroboré par le stagiaire lui-même qui nous explique éprouver énormément de difficultés à imposer son autorité aux élèves et à se faire respecter auprès d'eux.

A partir d'une étude antérieure (Vandercleyen et al., 2012), nous avons pu établir le « profil émotionnel » de Jean. II se révèle être un stagiaire très anxieux, qui a tendance à apprécier toute intervention à l'égard des élèves - que ce soit lors de consignes collectives ou individuelles - comme étant source de menace pour son bien-être. II explique ne pas oser intervenir de crainte de perdre le contrôle de la classe et de risquer d'altérer le climat relationnel établi entre les élèves et lui. II relate être complètement inhibé face aux élèves et incapable d'agir au moment opportun, malgré l'attitude respectueuse des élèves à son égard.

4. 1. 1. Une observation centrée sur l'évaluation des « manques » du stagiaire Fabienne utilise principalement un comportement d'évaluation lorsqu'elle observe le stagiaire. Elle a tendance à se centrer davantage sur ce qui ne va pas, sur ce qui manque au stagiaire pour être un «bon enseignant ». Elle est particulièrement critique concernant la (non) maîtrise des techniques de la communication. Par exemple, au sujet de la capacité du stagiaire à capter l'attention des élèves: "Tu vois, là il intervient mais personne n'écoute ! Ce n'est pas la première fois, ça ne va pas ! ». Selon la maître de stage, Jean n'est pas assez directif dans ses consignes : "II n'est pas assez strict... II est trop gentil! » (MSJ50). Cependant, Fabienne relativise ses propos, en précisant que ce genre de difficultés est rencontré par la plupart des stagiaires : "C'est vrai que ce n'est pas évident au début quand tu enseignes de ne pas être trop préoccupé par la gestion de classe. Lui, il est seulement préoccupé par le fait qu'ils travaillent. II a l'impression que cela bouge et il est content, mais après, pour rendre l'enseignement qualitatif, c'est une autre histoire ! ». Principalement centrée sur les manques du stagiaire, elle exprime aussi de temps à autres ce qu'elle apprécie dans l'intervention de celui-ci, comme son enthousiasme et la qualité de sa relation avec les élèves: " J'aime bien quand il démontre avec tout le groupe, je trouve cela très chouette, parce que c'est la meilleure façon de donner envie aux élèves de participer... En plus, il a un bon contact avec les élèves. C'est bien, je trouve que c'est important! »(MSJ56). 


\section{eJRIEPS 28 janvier 2013}

Fabienne présente également la particularité d'exprimer ses émotions à maintes reprises pendant l'observation de la séance. A différents moments, elle partage ses propres doutes, ses inquiétudes, ses déceptions : "Je suis un peu déçue, je ne m'attendais pas du tout à cela. Parce que les autres séances, il avait été capable de s'ajuster, et il me semblait qu'il avait pigé ce que je lui demandais. Mais je n'ai peut-être pas assez répété, je n'ai peut-être pas assez noté. Je n'en sais rien, je devrais regarder!»(MSJ51). Fabienne montre par là sa capacité à remettre en question son accompagnement. Elle dit d'ailleurs se sentir en partie responsable de l'échec du stagiaire pour cette séance, en précisant qu'elle aurait dû davantage l'aider à se préparer. Souvent impliquée émotionnellement dans la séance, elle commente les actions du stagiaire en faisant comme si elle s'adressait à lui, mais sans que le stagiaire ne puisse entendre son commentaire : "Ils l'ont déjà travaillé, tu sais mon ami, le renforcement musculaire ! »(MSJ12). Ou parfois de manière plus directive : «Allez, corrige, corrige ! » (MSJ49).

4. 1. 2. Styles, comportements et objets de supervision de Fabienne

La figure 1 montre la répartition des trois styles de supervision dans l'ensemble du discours post-séance de Fabienne. Cette dernière a tendance à adopter un style de supervision plutôt « directif », puisque $58 \%$ de l'ensemble de son discours est représenté par ce style. Le style « démocratique » est également très présent (38\%). Le style « expérientiel », quant à lui, est minoritaire (4\%), comparativement aux deux autres.

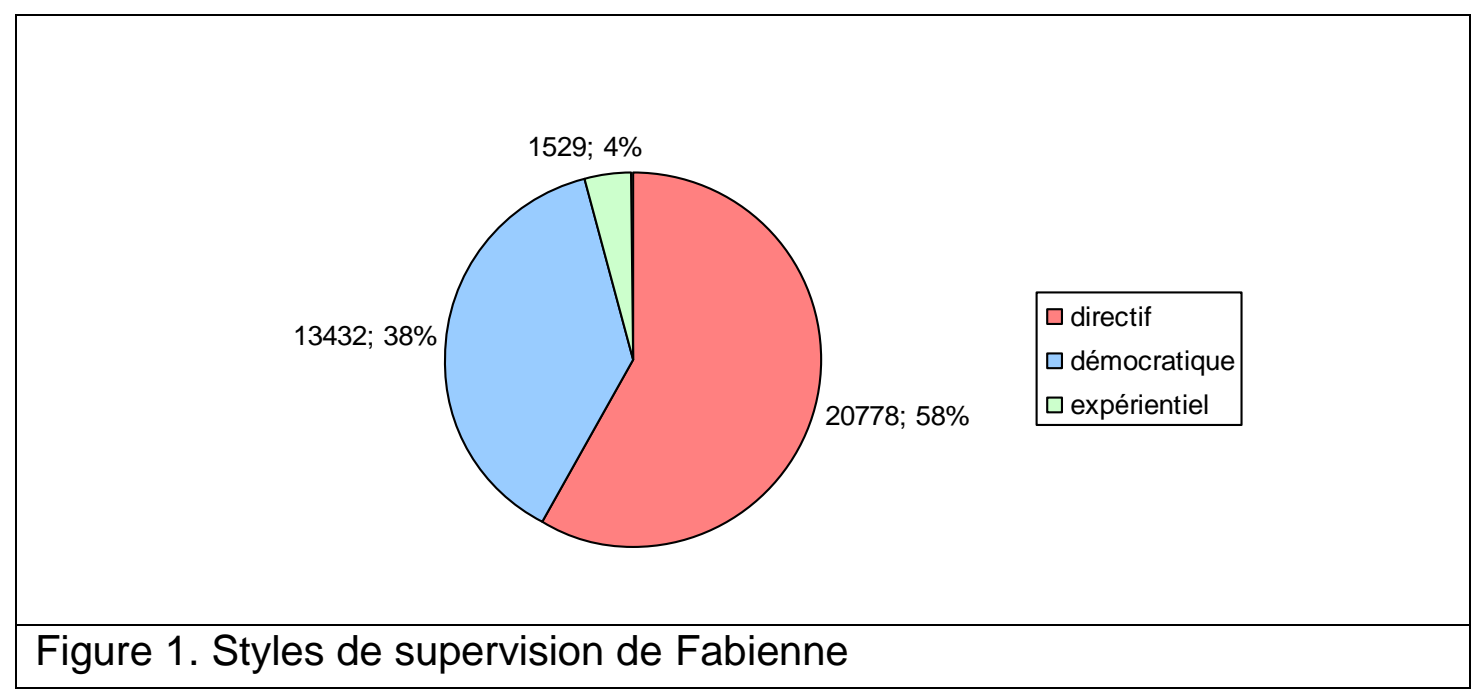

Comme illustré à a figure 2, l'instruction est le comportement directif de supervision le plus utilisé par MSJ. Celui-ci représente à lui seul plus d'un tiers du style directif. La sécurisation et la provocation - comportements directifs également - représentent un autre tiers. Si l'évaluation est très présente, elle ne forme, avec la direction, qu'une part 


\section{eJRIEPS 28 janvier 2013}

minoritaire du style directif. Ainsi, contrairement à ce que le graphe pourrait laisser supposer, il ne s'agit pas d'une maître de stage «tyrannique ». En ce sens, il est important de clairement distinguer les différents comportements de supervision repris sous le vocable « directif », pour se rendre compte qu'il ne s'agit pas uniquement de direction ou d'évaluation.

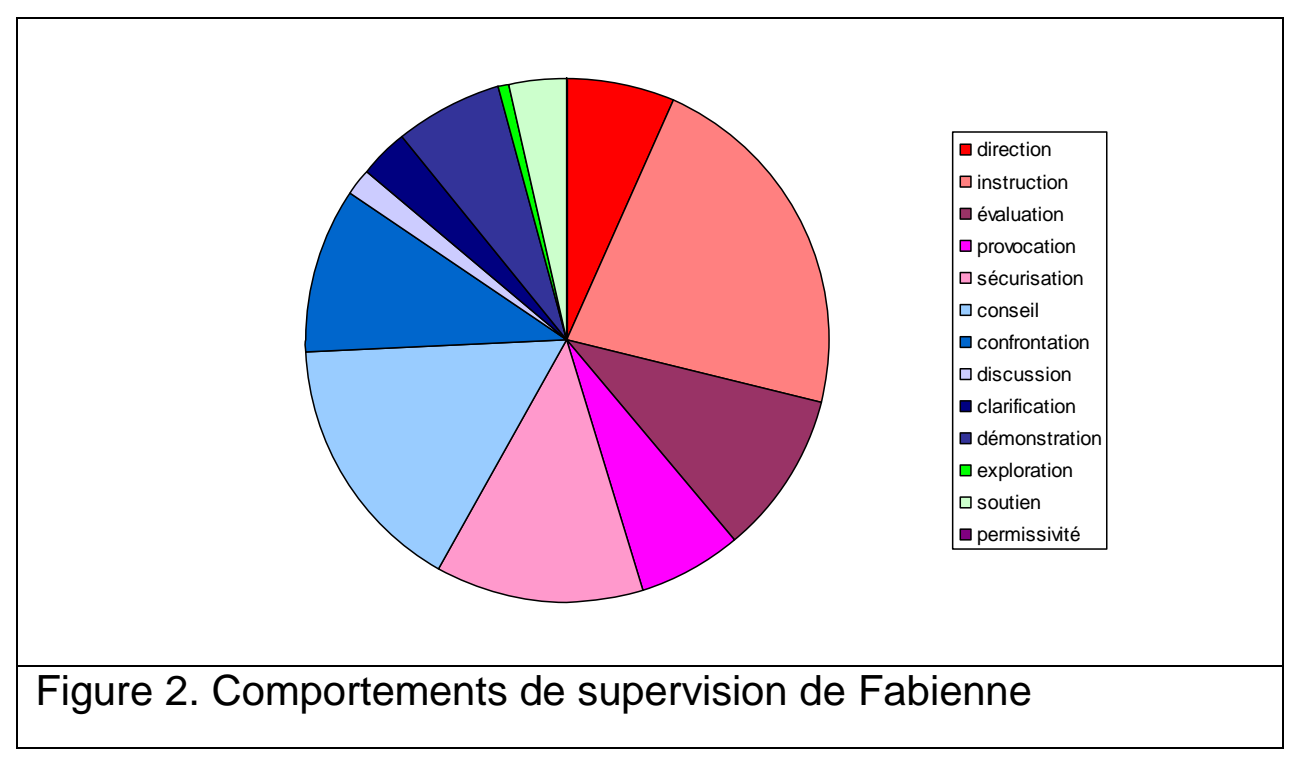

Concernant le style démocratique, cette figure révèle une maître de stage qui donne massivement des conseils au stagiaire. La confrontation est également très présente, autant que l'évaluation par exemple. Le comportement de démonstration est utilisé avec parcimonie. Bien que présents, les comportements de clarification et de discussion sont peu empruntés par Fabienne avec Jean. Le style «expérientiel » est principalement représenté par le comportement de soutien; l'exploration est minime, en termes de volume du moins. 


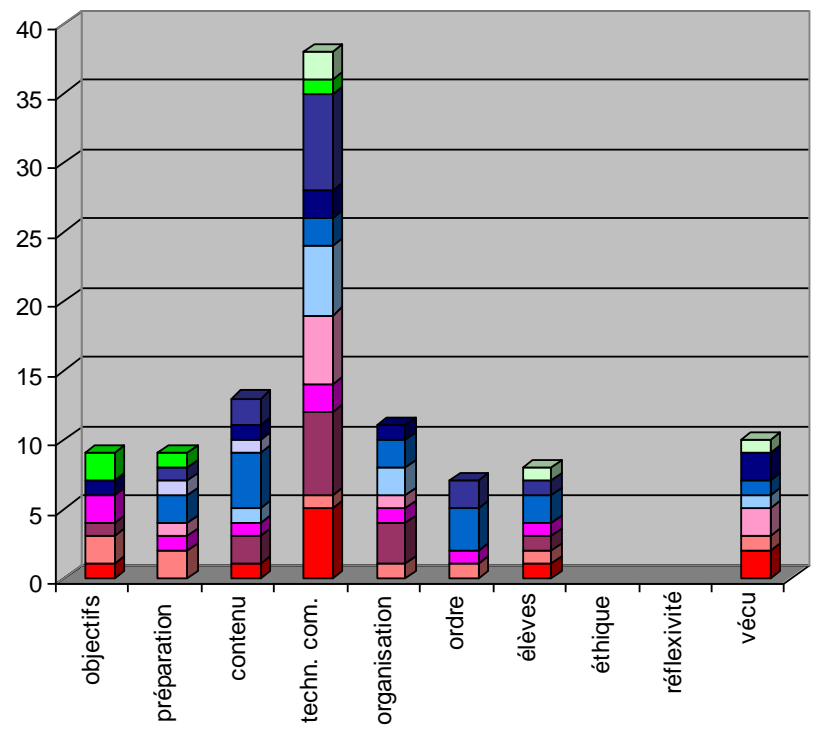

$\square$ permissivité

$\square$ soutien

$\square$ exploration

$\square$ démonstration

- clarification

$\square$ discussion

$\square$ confrontation

$\square$ conseil

$\square$ sécurisation

$\square$ provocation

$\square$ évaluation

$\square$ instruction

$\square$ direction

Figure 3. Objets de supervision en fonction des comportements de Fabienne

L'essentiel du discours (60\%) de Fabienne est centré sur les techniques de communications verbale et non verbale du stagiaire (cf. figure 3). Les quarante autres pourcents sont partagés de manière plus ou moins équitable entre les autres objets de formation, à l'exception de l'éthique et de la réflexivité, non représentées. Cette figure met également en évidence la répartition des différents comportements de supervision en fonction de chaque objet de formation. Nous avons fait le choix de ne présenter que l'objet le plus discuté (techniques de la communication) et le vécu du stagiaire, objet central de notre travail.

Si le style directif est dominant pour discuter des techniques de communication, la quasitotalité du panel de comportements de supervision possibles est utilisé par Fabienne. Par exemple, Fabienne évalue plutôt négativement la prise en main du stagiaire : « Je trouve ça très sympa que tu sois là pour accueillir les élèves mais par contre je trouve que ça manque vraiment fort d'efficacité (...) Mais tu vois ton attitude, moi je trouve que c'est plus un accueil de club de sport » (MSJ1, eval). Par le biais d'un comportement de direction, Fabienne se montre également intransigeante concernant la capacité du stagiaire à intervenir de manière autoritaire : «Non, là je ne suis pas d'accord, il faut y aller! II faut t'exprimer, c'est ton rôle ! Tu dois intervenir, tu es là pour ça ! » (MSJ4, dir). Par ailleurs, Fabienne n'hésite pas à exagérer ses impressions, dans le but de provoquer le stagiaire et l'inciter à «sortir de sa coquille »: «Moi j'ai eu l'impression que tu vivais un grand moment de solitude là, parce que on ne voit que toi au début de la séquence, là! On ne voit que toi tout seul et il n'y a personne autour de toi... " (MSJ2, prov). Cette attitude très 
« autoritaire » est cependant nuancée, équilibrée, par un discours rassurant de la part du maître de stage. La sécurisation est ainsi un comportement très présent chez Fabienne, en renvoyant à sa propre expérience : «Moi ça m'arrive encore de me rendre compte que je ne suis pas du tout sur la bonne voie et que je me plante et de changer carrément de direction! » (MSJ6, secu).

En marge de ce style dominant, Fabienne adopte également un style démocratique pour évoquer les habiletés d'enseignement que Jean pratique. Elle donne par exemple de nombreux conseils au stagiaire : "Par rapport à ça, tu pourrais te mettre au centre de la salle, mais dans l'axe du couloir, ce qui te permet d'observer à la fois la sortie des vestiaires, et ce qui se passe dans la salle, tu aurais une position plus stratégique ! » (MSJ7, cons). En utilisant la démonstration, Fabienne fait appel à sa propre expérience pour illustrer comment elle aurait résolu le problème rencontré par le stagiaire : «Quand je m’aperçois que quelque chose ne va pas, je commence par les arrêter. En général j'essaie de leur faire prendre conscience de ce qui ne va pas par le biais de l'humour »(MSJ8, demo).

De manière surprenante a priori, le vécu du stagiaire est évoqué de manière plutôt « directive ». Fabienne attache beaucoup d'importance à la prise en compte du « vécu émotionnel » du stagiaire lors du débriefing d'après séance. II s'agit d'ailleurs de l'objet le plus représenté dans son discours après le thème «techniques de communication ». Si les trois styles sont à nouveau présents, elle aborde le vécu en adoptant surtout un style directif. Face à l'anxiété du stagiaire et sa peur d'intervenir, elle tente de le recadrer avec une certaine fermeté : “C'est le souci aussi des élèves en technique d'expression parce qu'ils ont un cours comme ça avec moi... Le problème, c'est qu'ils se sentent toujours obligés de faire un truc! Je leur dis: «non, ça m'intéresse pas... Je veux simplement que tu sois là que tu habites ton corps et que si tu te sens bien ou si tu ne te sens pas bien, il faut l'exprimer! » (MSJ9, dir).

En adoptant un comportement d'instruction, elle explique de manière plus «théorique » ses raisons : «Moi ce que j'ai envie de te dire, c'est qu'il ne faut pas faire, mais être... Tout simplement ! Et à partir du moment où on est, avec nos soucis, nos peurs et des choses qui n'ont parfois rien à voir avec l'école ou avec ton cours, alors tu seras en phase avec toi-même ! II ne faut pas se cacher, pas paraître, mais être, exister, quoi ! Tu ne dois pas nécessairement faire quelque chose ! Ce n'est pas grave de rien faire, parfois ! Et si tu vois un truc, tu le commentes simplement ou à la limite tu ne dis rien... A un moment, il va 


\section{eJRIEPS 28 janvier 2013}

bien falloir quand même que tu interviennes, mais tu ne dois pas te tracasser de faire quelque chose à tout prix ! C'est ça que je veux que tu comprennes » (MSJ9, dir).

Son côté " directif » s'exprime aussi par la sécurisation dont elle fait preuve. Fabienne rassure ainsi à plusieurs reprises le stagiaire par rapport à ce qu'il vit « intérieurement » : «Tu as le droit de t'exprimer aussi et de dire qu'ils ne font pas ce que tu as demandé ! Mais il y'a aussi une différence entre le manque d'implication des élèves dans l'exercice, et le fait de ne pas le réussir ! S'ils essaient mais qu'ils n'y arrivent pas, c'est différent que de ne pas essayer ou de rien faire du tout ! Toi, ton rôle, c'est de faire en sorte qu'ils essaient » (MSJ18, secu).

Dans un style moins directif, Fabienne donne également des conseils à Jean sur la gestion des émotions : "Pour être, ça se travaille ! Par exemple travailler ton placement. Je te conseille d'apprendre à mieux te placer par rapport à l'atelier... Tu peux monter sur un banc par exemple pour avoir une vue plus surplombante... » (MSJ14, cons). Elle poursuit en lui démontrant comment faire : "Moi ça m'arrive de me mettre debout sur un banc pour voir de plus haut... A ta place, là, je me serais mise carrément debout sur le plinthe pour avoir une position plus dominante... En plus ça m'amuse encore bien ce genre de truc ! Après, chacun son style, mais moi ça me plaît et je crois que les élèves apprécient aussi ! »(MSJ15, demo). Une autre manière de Fabienne d'aider le stagiaire à faire face à ses émotions, c'est de le soutenir, en acceptant ses faiblesses : “C'est sans doute aussi dans ton tempérament, parce que c'est sûr qu'on ne peut pas changer comme ça du jour au lendemain ! Mais faut savoir que ça se travaille, ce n'est pas inéluctable... C'est plutôt rassurant, non ? » (MSJ45, sout).

\section{1. 3. Dynamique de supervision de Fabienne avec Jean}

Se dégage de l'entretien post-leçon, une dynamique de supervision récurrente chez Fabienne (cf. figure 4). Pour la plupart des objets de formation, la maître de stage commence systématiquement par évaluer les manques du stagiaire, avant de le sécuriser en relativisant par exemple ce qu'elle vient de dire. Ce passage de l'évaluation à la sécurisation passe, en fonction des objets évoqués, par des comportements de direction et/ou de provocation. Après avoir évalué et sécurisé le stagiaire, la maître de stage emprunte un style plus démocratique, en utilisant, en fonction des objets et de manière non exclusive, les comportements de conseil, de confrontation voire de démonstration. Enfin, pour certains objets (techniques de communication et vécu émotionnel), Fabienne termine son intervention par un comportement de soutien. 


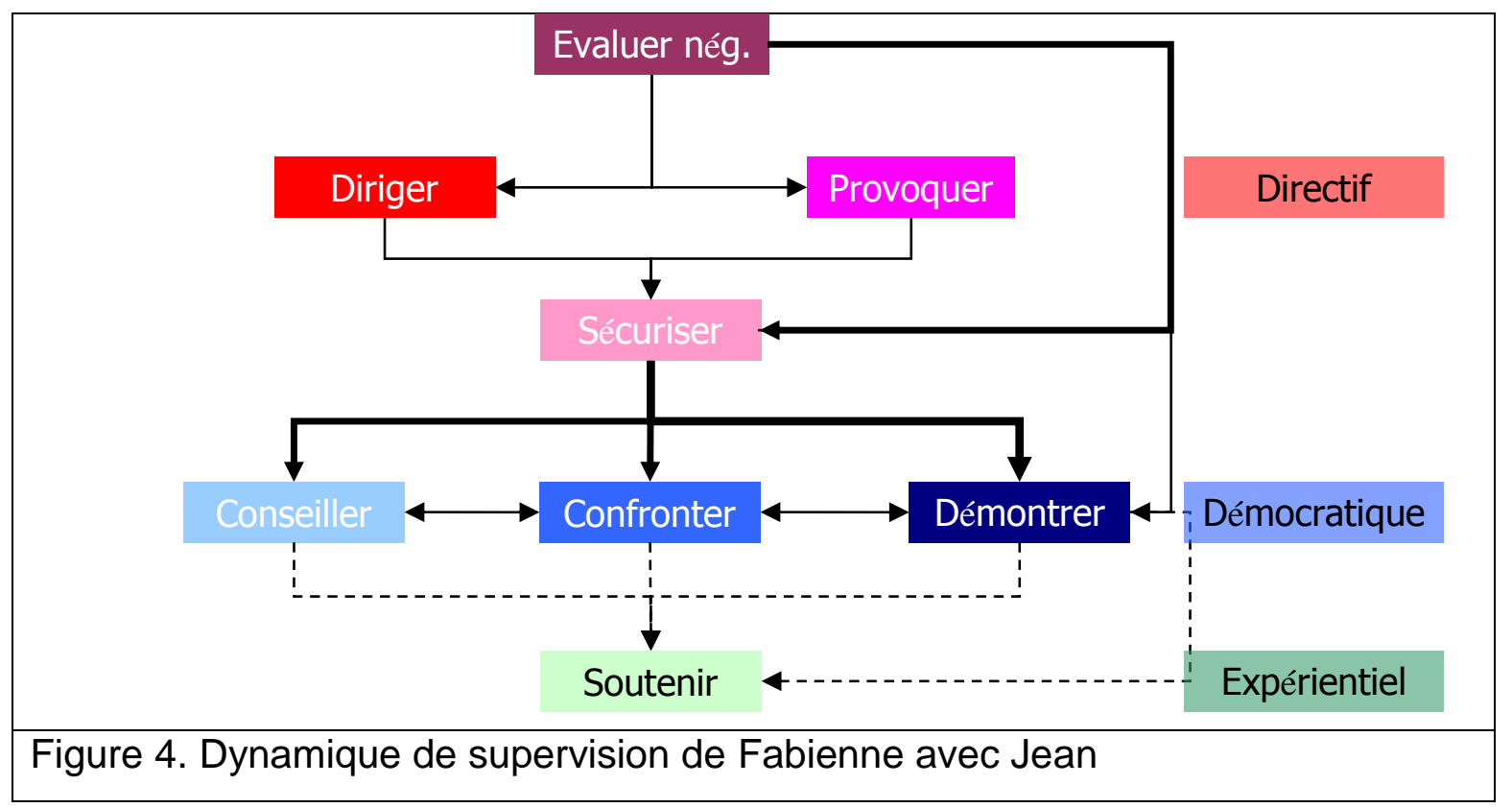

Globalement, Fabienne adopte un style de supervision plutôt directif, en utilisant principalement les comportements d'instruction et d'évaluation pour évoquer pratiquement tous les objets de formation. La sécurisation est également fortement empruntée, notamment lorsqu'elle parle de techniques de communication et du vécu personnel du stagiaire. Une des particularités de ce maître de stage est d'utiliser la direction avec parcimonie, mais de manière systématique néanmoins, quel que soit l'objet de formation discuté. Autre particularité : l'utilisation récurrente de la provocation. Fabienne adopte par ailleurs un style démocratique, par l'intermédiaire de conseils fréquents au stagiaire, portant principalement sur les techniques de communication, l'organisation et les contenus. La confrontation est un des comportements " démocratiques » les plus présents pour évoquer pratiquement tous les objets de formation. Fabienne utilise aussi la démonstration pour faire allusion à son expérience personnelle d'enseignante et illustrer comment résoudre certains problèmes rencontrés par le stagiaire. Concernant le style expérientiel (minoritaire), Fabienne se montre plutôt soutenante en ce qui concerne les techniques de communication et le vécu émotionnel du stagiaire.

Ce maître de stage-là avec ce stagiaire-là attache une importance toute particulière à interroger le vécu émotionnel de l'étudiant. En témoigne le volume important d'énoncés s'y rapportant. De manière a priori surprenante, elle se montre très directive pour évoquer ce vécu, à la fois par la sécurisation mais aussi par des comportements de direction et d'instruction. Cette analyse amène à qualifier le profil d'accompagnement de Fabienne comme une maître de stage à la fois stimulante et sécurisante. Ces deux qualificatifs, a priori contradictoires, apparaissent complémentaires. D'un côté, elle stimule le stagiaire, 


\section{eJRIEPS 28 janvier 2013}

en cherchant sans cesse à le faire réagir et à le sortir de sa relative passivité, notamment par ses nombreux comportements de confrontation, de clarification ou encore de provocation. D'un autre côté, elle le sécurise, par son attitude compréhensive voire empathique, laquelle s'exprime par les comportements de sécurisation et de soutien. Ce profil d'accompagnement typique peut être expliqué par un souci permanent de « restructuration » du stagiaire » (Loizon, 2006). Fabienne identifie le problème et aide le stagiaire à se poser des questions, tout en le rassurant et en légitimant les difficultés qu'il rencontre.

4. 2. Maxime, un maître de stage empathique et bienveillant

Le maître de stage et la stagiaire se connaissent, puisque Marie est une ex élève de l'établissement où travaille Maxime; ce dernier lui ayant donné cours. Le contexte de cette deuxième étude de cas concerne une séance portant sur l'apprentissage technique de la manchette au volley-ball, à des élèves de $4^{\mathrm{e}}$ secondaire. Ce thème est imposé à la stagiaire dans la mesure où la classe se trouve dans un cycle consacré à la manipulation de ballon. Marie affirme ne pas être spécialiste en sports collectifs, mais est très motivée malgré tout à l'idée d'enseigner cette matière. Elle nous dit avoir consacré beaucoup de temps et d'énergie à préparer sa séance.

Une étude préalable (Vandercleyen et al., 2012) a permis de caractériser le profil de Marie. Elle se révèle être une stagiaire plutôt discrète, voire introvertie. D'après Maxime, elle éprouve également des difficultés en termes de gestion de classe, notamment sur le plan de l'organisation spatiale du groupe et du matériel. La faible intensité de sa voix ne semble pas l'aider pour asseoir son autorité. Les émotions qu'elle vit sont variables et plutôt négatives, de l'ordre de la contrariété, de l'agacement voire de la frustration. A d'autres moments, elle dit ressentir une certaine forme de plaisir, très fugace, lorsqu'elle observe les élèves impliqués dans la tâche proposée.

4 .2. 1. Observations pendant la séance: évaluer, relativiser, anticiper, interpréter

Pendant la séance, Maxime évalue continuellement les comportements de la stagiaire. A l'instar de Fabienne, il est surtout centré sur les «manques » du stagiaire, dont principalement la qualité des techniques de communications. II lui reproche par exemple d'être peu motivante lors de ses consignes de lancement : "Donner un coup de sifflet et puis dire: "ok, c'est parti ! ", c’est infiniment moins motivant que de dire : "Qui sera le premier à arriver de l'autre côté ? Attention, prêt ? Allez, go, à fond ! ». Il faut mettre les élèves dans une situation de défi. Ça manque de piment. C'est beaucoup trop faible à 


\section{eJRIEPS 28 janvier 2013}

mon goût. Je trouve qu'elle doit travailler la qualité de ses relances! (MSM13). Cependant, conscient de la dominance de ses jugements à valence plutôt négative, Maxime se montre très autocritique. II s'efforce de voir le positif chez la stagiaire, même s'il avoue être spontanément plus attentif à « ce qui ne va pas ! »: " Je ne dis rien mais c'est parce que je me rends compte que les remarques que j'annote sont surtout des remarques négatives » (MSM30). Pour relativiser ses propos, il met par exemple en avant la qualité de la préparation de Marie : «A travers les exercices qu'elle propose, on peut apprécier la qualité de sa préparation » (MSM35).

Une des principales caractéristiques de ce maître de stage est qu'il anticipe fréquemment les comportements de la stagiaire. Ainsi, Maxime ne se contente pas de décrire les comportements de la stagiaire ou de les évaluer. II tente souvent d'imaginer ce qui pourrait se passer : “Un moment important aussi, ça sera de voir comment elle va s'y prendre pour clôturer la séance... Est-ce qu'elle va avouer aux élèves qu'elle avait prévu un match ? (MSM53). Une autre particularité de Maxime est d'interpréter les actions de Marie : "Je suis sûr qu'elle doit être déçue, mais si je la laisse, les élèves vont être en retard au cours suivant, et ça je ne peux pas me permettre ! » (MSM55). De même, il lui arrive de se mettre à la place de la stagiaire, afin de mieux cerner son possible ressenti : " $A$ mon avis, elle est dans son trip, elle est en train de revivre sa séance et de repenser sans doute à ce jeu qui n'a pas été... On lui posera la question tout à l'heure ! » (MSM57). Si le maître de stage émet des hypothèses d'action, il reste cependant très prudent et évite de juger la stagiaire avant de l'avoir interrogée. En résumé, Maxime adopte, un style de supervision très directif pendant la séance, principalement représenté par un comportement d'évaluation. Conscient de ce jugement permanent, il s'efforce de mettre en évidence également les points positifs de l'intervention de Marie. Enfin, Maxime présente la double caractéristique d'anticiper et d'interpréter les actions menées par la stagiaire.

4. 2. 2. Styles, comportements et objets de supervision de Maxime après la séance

Lors de son discours d'après séance, Maxime présente un profil mixte, plutôt équilibré (cf. figure 5). Les styles démocratique et directif se retrouvent dans une proportion identique, tandis que le style expérientiel est relativement bien représenté. 


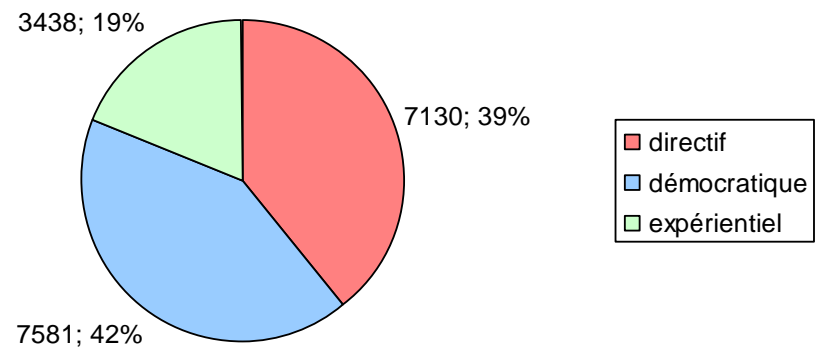

Figure 5. Styles de supervision de Maxime (MSM)

Plus précisément, la figure 6 (ci-dessous) met en évidence des comportements d'instruction et d'évaluation très présents pour expliquer le style directif de Maxime. Ainsi, si le maître de stage de Marie peut être considéré comme étant plutôt directif, le comportement de direction est néanmoins très peu utilisé. Concernant le style "démocratique ", Maxime est surtout dans la confrontation et dans la discussion. Les comportements de conseil et de clarification sont également présents mais représentent une part infime des comportements démocratiques. Le soutien et l'exploration sont les deux comportements adoptés par ce maître de stage dans un style «expérientiel ».

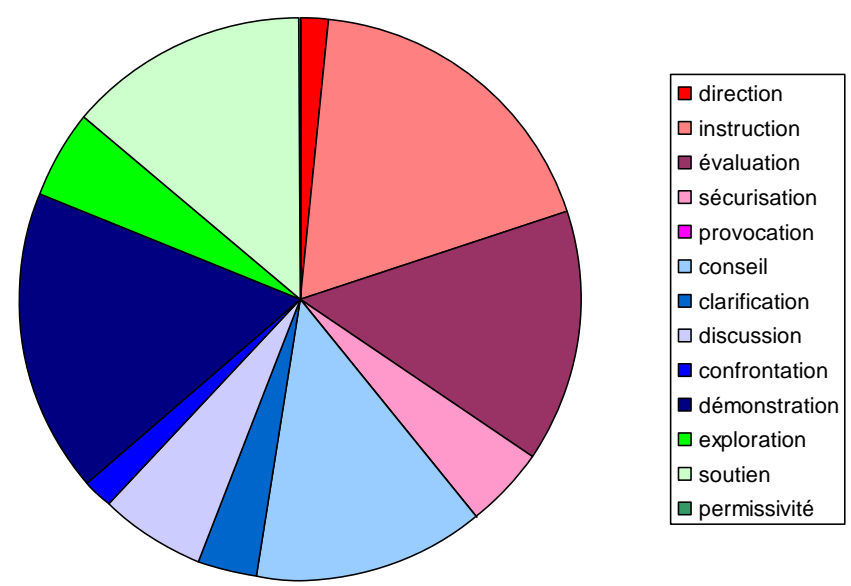

Figure 6. Comportements de supervision de Maxime (MSM)

Concernant les objets de formation discutés par Maxime (illustrés à la figure 7), il est intéressant de constater que la plupart sont discutés autant dans un style démocratique que directif. Les techniques de communication sont clairement l'objet le plus régulièrement 


\section{eJRIEPS 28 janvier 2013}

abordé. Bien que secondaire, le vécu personnel de la stagiaire est présent dans le discours post-séance de Maxime.

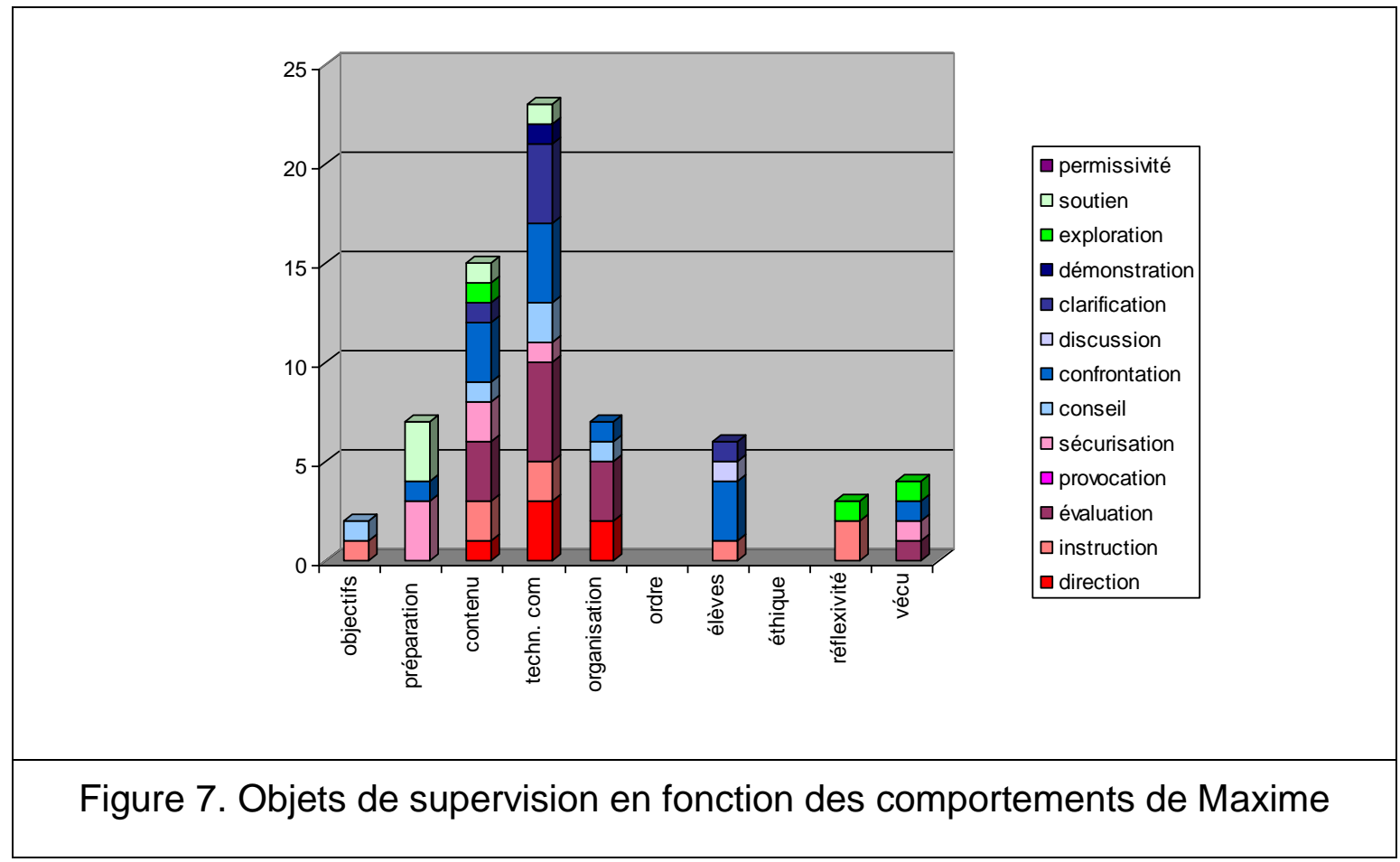

De la même façon que pour Fabienne, la dimension "technique de communication" occupe une place très importante (plus de 50\%) dans le discours d'après-leçon de Maxime. Le style directif est principalement utilisé pour aborder cet objet de formation. Par un comportement d'instruction, ce maître de stage passe beaucoup de temps à expliquer à la stagiaire certains principes fondamentaux : "On a tendance, et c'est spontané, à être centré sur l'action qui se passe devant nos yeux, et à côté de ça il se passe plein d'autres choses, pour lesquelles on n'est pas nécessairement vigilant! »(MSM1, instr). A plusieurs reprises, Maxime structure et dirige la stagiaire sur la manière de s'y prendre pour améliorer sa technique de communication : "Il faut être plus directive dans tes consignes, ça peut t'aider, de manière à ce que ça se mette en place beaucoup plus vite " (MSM2, dir). En même temps, II rassure beaucoup la stagiaire sur cet objet : "C'est une difficulté bien légitime, de ressortir de l'action qui est en train de se passer pour prendre du recul et voir ce qui se passe dans l'ensemble " (MSM3, secu).

Dans un style davantage " démocratique ", Maxime confronte ses perceptions avec celles de la stagiaire : "Si tu étais plus directive, ou si tu les désignes, ça prête peut-être moins à confusion, tu ne crois pas ? Qu'en penses-tu ? " (MSM6, conf). II donne çà et là quelques conseils également sur le plan technique de communication : "Et donc pour 


\section{eJRIEPS 28 janvier 2013}

mieux les voir, tu devrais pour ça prendre plus de recul, te mettre plus à distance de l'action! » (MSM8, cons). Rarement, il lui arrive d'évoquer sa propre manière de faire : « Je pense qu'en posant ma voix différemment de toi, je garde une distance avec les élèves » (MSM10, demo). II cherche plutôt à clarifier au mieux ce qu'il s'est réellement passé en sollicitant à plusieurs reprises le point de vue de la stagiaire : «Autre chose qui m'a interpellé, c'est que tu regardes à droite, mais jamais sur ta gauche... c'est peut-être parce qu'on était sur ta gauche et que tu ne voulais pas faire face à la caméra ? Ton avis ? " (MSM12, clarif), plutôt que de faire référence systématiquement à son expérience d'enseignant. Dans un style expérientiel, Maxime se montre également très soutenant malgré la non maîtrise des techniques de communication. II n'hésite pas, dès que l'occasion se présente, à valoriser la stagiaire : "Je crois aussi que ça aurait été une bonne solution! » (MSM14, sout). De plus, Maxime montre clairement à plusieurs reprises son intention d'aider Marie à trouver les solutions elle-même aux problèmes qu'elle rencontre, en utilisant l'exploration : «On voit que tu les as placés en fer à cheval autour de toi, cette disposition-là présente des avantages et des inconvénients... qu'est-ce que tu verrais comme avantages ? »(MSM21, expl).

Par ailleurs, Maxime s'intéresse fortement au vécu émotionnel de Marie. II utilise les trois styles de supervision pour en parler. II évalue notamment de manière positive la manière avec laquelle Marie ose partager son vécu avec les élèves : «C'est bien de leur partager sincèrement, en toute simplicité, ton ressenti à la fin de la séance » (MSM17, eval). II tente également de la rassurer par la sécurisation : “Tu as le droit à l'erreur ! Tu ne peux pas non plus penser à tout... » (MSM15, secu). Dans un style plus démocratique, il confronte ses impressions avec celles de la stagiaire : "Je crois qu'à ce niveau-là les élèves sont assez coopérants, Enfin, je ne sais pas comment tu l'as vécu ? » (MSM7, conf).

\section{2. 3. Dynamique de supervision de Maxime}

Aucune dynamique récurrente d'un incident à l'autre n'émerge dans le discours post-leçon de Maxime. Cependant, on peut constater au moins trois comportements distincts appartenant à un gradient différent sur le continuum de directivité - qui apparaissent indépendamment de l'objet de supervision: l'instruction (directif), la confrontation (démocratique) et le soutien ou l'exploration (expérientiel). L'analyse montre par ailleurs un maître de stage qui est dans l'interaction, dans l'échange, avec la stagiaire qu'il accompagne. Les nombreux comportements de confrontation et de discussion démontrent son intention d'initier le dialogue et sa volonté d'échanger son point de vue de manière constructive avec la stagiaire. Dans tous les cas, Maxime montre qu'il s'intéresse 


\section{eJRIEPS 28 janvier 2013}

principalement au ressenti de la stagiaire. II ne fait d'ailleurs que très rarement allusion à son expérience personnelle d'enseignant.

Maxime montre un profil d'accompagnement plutôt équilibré entre les trois styles définis par Brûlé (1983). Le style directif est principalement expliqué par un comportement d'instruction très présent, de même que celui d'évaluation. Une particularité de ce maître de stage est d'être constamment dans l'interaction, le dialogue et l'échange de points de vue avec la stagiaire. Cette caractéristique est prégnante au vu des comportements de confrontation et de discussion régulièrement exploités. Son style démocratique se distingue également par un comportement de démonstration quasi absent, ne faisant que très rarement appel à son expérience personnelle pour illustrer ses propos. Son attitude très soutenante dans un style expérientiel bien présent constitue une autre caractéristique du maître de stage de Marie, très centré sur les préoccupations de la stagiaire. II propose une relation d'aide basée sur l'empathie, la bienveillance et le dialogue constructif. Cette « compréhension sans complaisance » (Donnay \& Charlier, 2006) est caractéristique du profil de supervision de Maxime avec Marie.

\section{Discussion}

Après avoir identifié les singularités émanant des pratiques d'accompagnement des deux maîtres de stage, les paragraphes qui suivent visent à mettre en évidence les invariants issus de cette double étude de cas contrastée, à la lumière de la littérature.

5. 1. Accompagner pour aider à « apprendre à enseigner »

Chez Fabienne comme chez Maxime, l'omniprésence de comportements de supervision directifs montre deux maîtres de stage qui cherchent à cadrer leur stagiaire en manque de repères. Empathique, Fabienne accompagne Jean dans son développement professionnel, en stimulant notamment sa réflexion sur l'action. Elle l'aide à identifier le problème et à se poser des questions, tout en le rassurant et en légitimant les difficultés qu'il rencontre. Cette attitude, à la fois stimulante et sécurisante, sur un mode davantage compréhensif que persuasif, est tout à fait caractéristique du profil d'accompagnement de Fabienne. Loin de se targuer d'être un modèle pour le stagiaire, elle prône plutôt un «accompagnement réflexif » (Donnay et coll., 2001). Elle aide Jean à « apprendre à enseigner » (Chaliès \& Durand, 2000). Si elle donne au stagiaire des conseils utiles « ici et maintenant », elle lui fournit aussi des pistes valables « ailleurs et plus tard (FeimanNemser, 1996). L'exemple de la formation en techniques d'expression qu'elle propose au stagiaire est tout à fait éloquent à cet égard. Par ailleurs, la faible présence du 


\section{eJRIEPS 28 janvier 2013}

comportement de démonstration, particulièrement chez Maxime, montre deux maîtres de stage qui évitent de mettre en avant leur propre expérience d'enseignant, au profit du vécu du stagiaire.

5. 2. D'une posture de praticien à celle de formateur

L'analyse montre que les deux maîtres de stage oscillent entre une attitude de « savoirs à transmettre » et celle d'un compagnon inducteur d'action et de réflexion. « Le dosage des conduites formatrices du maître de stage sera fonction de sa perception des potentialités de développement autonome du stagiaire, pas encore collègue ni plus tout à fait élève " (Donnay et al., 2001, p.34). Pour le maître de stage, ce changement de posture passe inévitablement par une analyse de sa propre pratique et une prise de distance de ses propres modèles d'action. «Un pré requis incontournable au compagnonnage réflexif est d'être soi-même un praticien réflexif » (Donnay et al., 2001, p.38). Aider le stagiaire à réfléchir sur lui-même et sur ses propres pratiques implique que le maître de stage luimême soi capable de réflexivité sur soi et sur ses pratiques. Cette compétence métacognitive d'explicitation et d'analyse constitue un socle, un prérequis du formateur de terrain. Alors que la réflexion met des mots sur les actions dans la situation vécue et l'interprète déjà, la réflexivité fait adopter une posture d'extériorité, de réflexion sur la réflexion (métacognition), pour déconstruire la situation pour mieux reconstruire l'objet d'analyse à un autre niveau. En ce sens, la réflexivité met le praticien sur une trajectoire de théorisation, de construction de ses connaissances pratiques (Donnay \& Charlier, 2006) Si le maître de stage est idéalement placé pour induire la réflexivité du stagiaire, encore faut-il qu'il ait appris comment y arriver !

Selon Donnay et Charlier (2006), cette réflexivité ne va pas de soi, elle doit être induite par un tiers. II s'agit de placer le stagiaire dans une attitude de recul par rapport à soi et de mise à distance de la situation vécue pour pouvoir en extraire le sens et lui faire s'approprier un langage professionnel. Cette posture requiert de la part du maître de stage un travail sur soi, de gestion de sa propre subjectivité, et le développement de techniques d'écoute active, d'observation, d'objectivation du réel, d'intransigeance par rapport à l'éthique professionnelle, dans une perspective d'accompagnement au développement professionnel du stagiaire. A ce sujet, certains passages issus du discours de Fabienne après la séance montrent qu'elle est consciente que son savoir n'en constitue qu'un parmi les possibles («tu pourrais faire comme ceci...») et qu'elle se rend disponible pour aider le stagiaire à se construire professionnellement. II en va de même pour Maxime qui dit à plusieurs reprises « ne pas détenir la vérité », mais que seule la stagiaire connaît la 


\section{eJRIEPS 28 janvier 2013}

solution. II remet également en question à plusieurs reprises sa manière d'accompagner, tant pendant la séance, qu'après.

5. 3. La « compréhension sans complaisance » : une compétence relationnelle La capacité des deux maîtres de stage à jongler avec un large panel de comportements de supervision révèle une certaine aisance de leur part à gérer l'interaction. Leur capacité à écouter le vécu du stagiaire, à le rassurer, à l'encourager autant qu'à le conseiller au moment opportun, à collaborer avec lui sous la forme d'échanges de points de vue, à le bousculer dans ses conceptions et dans sa pratique, constituent autant d'indicateurs indéniables de compétences relationnelles et de communication. Ils offrent un accompagnement «à géométrie variable » (Loizon, 2006), oscillant, suivant les incidents et/ou les objets abordés, entre le pôle «transmission » des ficelles du métier, et le pôle «autonomisation » en stimulant la construction de pratiques singulières par le stagiaire et le développement d'une identité professionnelle forte. Cette compétence relationnelle socio-affective - exige du maître de stage de reconnaitre et de gérer sa propre subjectivité avant d'aider le stagiaire à gérer la sienne.

Pour Paul (2004), gérer sa propre subjectivité suppose notamment de renvoyer au stagiaire des questions «non chargées », c'est-à-dire sans jugement implicite sur la personne, pour faire émerger les implicites des actes du stagiaire. Concrètement, cette attitude compréhensive sans complaisance passe par aider le stagiaire à :

- reconnaître le vécu de ses émotions dans le respect des élèves ;

- objectiver le réel (accepter le choc du réel, distinguer le « réel » du « souhaité »...) ;

- envisager d'autres possibles.

Les résultats issus de cette double étude de cas montrent deux maîtres de stage qui tendent vers cette attitude. En effet, à plusieurs reprises au cours de l'entretien post-leçon, les deux maîtres de stage s'intéressent au vécu du stagiaire. Sans mettre de côté leurs propres émotions - vécues notamment pendant la séance -, Fabienne par exemple s'attache à se focaliser sur les préoccupations du stagiaire. Cependant, les résultats montrent aussi que ce maître de stage utilise l'instruction et le conseil comme réponses aux sollicitations importantes du stagiaire sur le plan émotionnel. Autrement dit, pour répondre aux difficultés affectives soulevées par Jean, elle renvoie parfois à des comportements plutôt directifs (instruction, conseils), et non nécessairement expérientiels ou réflexifs. 


\section{eJRIEPS 28 janvier 2013}

5. 4. Prendre en compte la subjectivité du stagiaire par l'analyse de pratique

Tant Fabienne que Maxime incitent leurs stagiaires respectifs à se confronter à la situation réelle, en les raccrochant aux faits vécus. Ils les aident à évoquer ces situations, à les décrire, à les expliciter. Par exemple, Maxime aide Marie à analyser les situations dans le temps et dans l'espace classe, ainsi qu'à mettre en rapport les événements en fonction de relations causales. II l'aide également à envisager et à explorer d'autres possibles. En l'aidant ainsi à expliquer et à comprendre ce qui s'est joué dans la situation, Maxime applique - consciemment ou non - les principes fondamentaux de l'analyse de pratique. Loizon (2006) précise que la subjectivation du réel du stagiaire a tendance à, plus ou moins consciemment, lui occulter la part du réel qui ne lui conviendrait pas. Or, la tentation est grande de donner son point de vue sur l'interaction stagiaire-réel. Cette attitude n'est pas sans risque pour le maître de stage de projeter sa propre subjectivité dans cette interaction. "C'est le réel, même construit, qui doit rester la référence et qui sera formateur lorsque le stagiaire sera plus tard seul dans sa classe » (Donnay et coll., 2001, p. 41). Par son attitude bienveillante, Maxime adopte également un comportement très soutenant, très empathique, en veillant à garantir un climat de confiance avec la stagiaire. "Soutenir le stagiaire, avec compréhension mais sans complaisance par rapport aux réalités rencontrées, aide le stagiaire à apprendre sa profession dans un cadre sécurisé » (Paul, 2004, p.34).

Les deux maîtres de stage démontrent certaines qualités de médiation, en facilitant çà et là l'expression du vécu de la stagiaire ainsi que la décentration et la mise à distance «actes-personnes ». Cette capacité à décoder le décalage entre le discours du stagiaire et le réel sur lequel on lui ouvre les yeux n'est pas donnée à tout le monde. On retrouve par exemple chez Maxime la double compétence spécifique du formateur - qui le différencie de l'enseignant - relevée par Dugal (2008), à savoir d'une part la maitrise de l'analyse de pratique et d'autre part l'accompagnement par la médiation. A l'instar de Fabienne, l'attention de Maxime semble tournée sur le développement professionnel du stagiaire, en l'aidant notamment à se construire une identité professionnelle forte.

\section{Conclusions}

A la lumière du modèle de Brûlé (1983), cette recherche tente de caractériser les pratiques d'accompagnement des maîtres de stage lors d'un entretien post-leçon, à partir de deux études de cas contrastées. Par la mise en évidence de leurs objets, de leurs comportements et de leurs styles de supervision, nous souhaitons comprendre comment 


\section{eJRIEPS 28 janvier 2013}

ces maîtres de stage s'y prennent pour prendre en compte le vécu personnel de leur stagiaire.

L'analyse des données montre que les deux maîtres de stage impliqués dans cette étude adoptent un profil d'accompagnement « mixte » avec leur stagiaire, c'est-à-dire composé d'une combinaison de plusieurs styles de supervision. Chaque maître de stage présente une répartition singulière des trois styles de supervision: directif, démocratique et expérientiel. Au-delà des singularités, certains invariants peuvent être pointés. De manière générale, le style « directif » est clairement dominant chez chacun d'eux. Les deux comportements directifs les plus répandus sont «l'instruction » et «l'évaluation », bien que le comportement de « sécurisation » soit également très présent. Ces comportements majoritairement directifs s'expliquent par le profil de deux stagiaires en difficulté sur le plan de la gestion de classe et, de manière plus globale, au niveau de la gestion des émotions. Le style démocratique est marqué par un comportement de « conseil » omniprésent, alors que la «démonstration» (référence à sa propre expérience d'enseignant) est peu présente. Le style « expérientiel », principalement représenté par un comportement de « soutien », est plus discret.

Par ailleurs, les résultats montrent que le vécu émotionnel du stagiaire n'est pas (ou peu) abordé de manière frontale ou directe par chacun des maîtres de stage. Ils ont tendance à y faire référence de manière indirecte ou détournée, en mettant l'accent sur d'autres objets de formation également sources d'émotions. Cette attention indirecte portée au ressenti des stagiaires peut les aider à gérer leurs émotions. Cette interprétation est discutée en relevant les singularités de chacun des maîtres de stage à prendre en compte les émotions de leurs stagiaires respectifs.

Enfin, à l'instar de l'étude de Portelance et al. (2009), la présente recherche suggère la mise en place d'une formation spécifique à l'égard des maîtres de stage concernant la posture d'accompagnement. Un des objectifs serait de les inciter à considérer le vécu émotionnel des stagiaires comme vecteur de développement professionnel (Goigoux \& al., 2009).

\section{Bibliographie}

Albarello, L., Bourgeois, E., \& Guyot, J-L. (2007). Statistique descriptive. Bruxelles: De Boeck. 


\section{eJRIEPS 28 janvier 2013}

Altet, M., Paquay, L., \& Perrenoud, P. (2002). Formateurs d'enseignants: quelle professionnalisation ? Bruxelles: De Boeck.

Boudreau, P. (2001). Que se passe-t-il dans un stage réussi ? Revue des Sciences de l'Education, 27(1), 65-84.

Boudreau, P. (2009). Pour un modèle de supervision de type inductif en formation des maîtres de stage en éducation physique. Éducation et francophonie, 37(1), 121 139.

Boutet, M., \& Rousseau, N. (2002). Les enjeux de la supervision pédagogique des stages. Québec : PUQ.

Bujold, N. (2002). La supervision pédagogique: vue d'ensemble. In M. Boutet \& N. Rousseau (Eds.), Les enjeux de la supervision pédagogique des stages. Québec : PUQ. 9-22.

Brûlé, P. (1983). Mesure du style de supervision : théorie et application. Chicoutimi : Ed. G. Morin.

Brunelle, J., Coulibaly, A., Brunelle, J-P., Martel, D., \& Spallanzani, C. (1991). La supervision pédagogique. Revue EP.S, 227, 58-64.

Carlier, G. (2002). Superviser des stagiaires en éducation physique : balises pour une fonction en voie de professionnalisation. Avante, 8(1), 96-111.

Carlier, G. (2009). Accompagner et former des maîtres de stage en éducation physique. L'expérience de l'Université catholique de Louvain. Éducation et francophonie, $37(1), 68-88$.

Chaliès, S., \& Durand, M. (2000). L'utilité discutée du tutorat en formation initiale des enseignants. Recherche \& Formation, 35, 145-180.

Chaliès, S., \& Raymond, J. (2008). Conseiller sur le métier : une nécessaire reconstruction du métier. Revue EP.S., 330, 53-70.

Desbiens, J.-F., Borges, C., \& Spallanzani, C. (2009). La supervision pédagogique en enseignement de l'éducation physique. Éducation et francophonie, 37(1).

Donnay, J., \& Charlier, E. (2006). Apprendre par l'analyse des pratiques. Initiation au compagnonnage réflexif. Namur : Presses Universitaires de Namur.

Donnay, J., Dejean, K., \& Charlier, E. (2001). Recherche sur le rôle des maîtres de stage dans la formation initiale des enseignants en Belgique francophone. Rapport de recherche FUNDP - Hautes-Écoles (non-publié). 


\section{eJRIEPS 28 janvier 2013}

Dugal, J-P. (2008). Le conseil en formation initiale des enseignants : intérêts et fonction des savoirs didactiques pour le tutorat des professeurs stagiaires en EPS. eJRIEPS, 14, 7-21.

Feiman-Nemser, S. (1996). Inventing a new role for cooperating teachers. Michigan: East Lansing.

Goigoux, R., Ria, L., \& Toczek-Capelle, M-C. (2009). Les parcours de formation des enseignants débutants. Clermont-Ferrand : Presses universitaires Blaise-Pascal.

Herbert, E., \& Worthy, T. (2001). Does the first year of teaching have to be a bad one? A case study of success. Teaching and Teacher Education, 17, 897-911.

Leriche, J., Desbiens, J.-F., Dugal, J.-P., \& Amade-Escot, C. (2010). Analyse de l'accompagnement du stage en responsabilité au Québec et en France : un regard sur les entretiens post-leçons à l'aide de l'écologie de la classe. eJRIEPS, 19(1), 71-99.

Loizon, D. (2006). Analyse de pratiques : miroir des apprentissages. Des outils pour la formation et l'enseignement en EPS. Dijon : CDRP de Bourgogne.

Molina, E., \& Gervais, C. (2008). Les stages en formation à l'enseignement : pratiques et perspectives théoriques. Québec: PUQ.

Mucchielli, R. (1988). L'analyse de contenu. Paris: ESF.

Paul, E. (2004). L'accompagnement: une posture professionnelle spécifique. Paris: L'Harmattan.

Paul, E. (2009). Autour du mot « accompagnement». Recherche et Formation, 62, 91 107.

Portelance, L. (2009). Elaboration d'un cadre de référence pour la formation des enseignants-associés québécois. Éducation et francophonie, 37(1), 26-49.

Raymond, D., \& Lenoir, Y. (1998). Enseignants de métier et formation initiale. Bruxelles: De Boeck.

Ria, L., Sève, C., Saury, J., \& Durand M. (2003). Beginning teachers' situated emotions: a study of first classroom experiences. Journal of Education for Teaching, 29(3), 219-234.

Robo, P. (2009). La formation des enseignants: l'accompagnement en formation professionnelle. Journées d'étude du Pôle Nord-Est, Conférence d'ouverture. 2930 janvier. Dijon : IUFM de Bourgogne.

Saint-Arnaud, Y. (2003). L'interaction professionnelle : efficacité et coopération. Montréal : Presses de l'université de Montréal. 


\section{eJRIEPS 28 janvier 2013}

Serres, G., Ria, L., Adé, D., \& Sève, C. (2006). Apprend-on vraiment à intervenir en formation initiale ? Prémisses du développement de l'activité professionnelle dans les dispositifs en alternance. Revue STAPS, 72, 9-20.

Theureau, J. (1992). Le cours d'action : analyse sémiologique : essai d'une anthropologie cognitive située. Berne : Peter Lang.

Towsend, T., \& Bates, R. (2007). Handbook of teacher education: globalization, standards and professionalism in times of change. Netherlands : Springer.

Van der Maren, J-M. (1995). Méthodes de recherche pour l'éducation. Montréal: PUM.

Vandercleyen, F. (2010). Gérer un incident critique organisationnel en éducation physique: régulation émotionnelle d'enseignants-stagiaire et accompagnement par leurs maîtres de stage. Thèse de doctorat en Sciences de la Motricité, UCL, Louvain-la-Neuve (non publiée).

Vandercleyen, F., Delens, C., Carlier, G., \& Boudreau, P. Preservice teachers in PE involved in an organizational critical incident: emotions, appraising and coping strategies. Physical Education \& Sports Pedagogy (accepted $5^{\text {th }}$ september 2012).

Yin, R., K. (1998). Case Study Research: design and methods. Beverly Hills: Sage Publications.

\section{Liste des acronymes}

APS : Activités physiques et sportives

ATR : Appui tendu renversé

CP : Conseiller pédagogique

MS : Maître de stage

MSJ : Maître de stage de Jean

MSM : Maître de stage de Marie

UCL : Université catholique de Louvain 
Annexes

\begin{tabular}{|c|c|c|}
\hline \multicolumn{3}{|c|}{ Annexe 1 - Définition des 13 catégories « comportements de supervision » (adapté de Brûlé, } \\
\hline \multirow{5}{*}{ 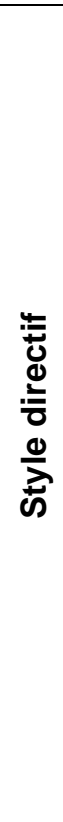 } & Direction (dir) & $\begin{array}{l}\text { Consiste à faire des recommandations en donnant des } \\
\text { indications précises ou des prescriptions, en imposant son } \\
\text { avis au stagiaire. } \\
\text { Ex. : «Tu dois faire ceci... ! »; «Fais cela ! » }\end{array}$ \\
\hline & Instruction (inst) & $\begin{array}{l}\text { Consiste à transmettre des savoir, savoir faire ou savoir être } \\
\text { sous la forme d'explications générales, pratiques ou } \\
\text { théoriques. } \\
\text { Ex.: «En général, il faut faire ceci...»; « Faire cela parce } \\
\text { que... » }\end{array}$ \\
\hline & Evaluation (eval) & $\begin{array}{l}\text { Consiste à porter un jugement critique, positif ou négatif, sur } \\
\text { l'efficacité des interventions ou des comportements du } \\
\text { stagiaire. } \\
\text { Ex.: «C'est dommage que... »; « Je trouve que... » }\end{array}$ \\
\hline & Sécurisation (secu) & $\begin{array}{l}\text { Consiste à rassurer le stagiaire dans ses appréhensions en } \\
\text { l'aidant à relativiser les difficultés qu'il rencontre. } \\
\text { Ex. : « Ne t'inquiète pas...!! ; « C'est normal...! » }\end{array}$ \\
\hline & Provocation (prov) & $\begin{array}{l}\text { Consiste à exagérer voire caricaturer volontairement certains } \\
\text { comportements pour forcer le stagiaire à réagir. } \\
\text { Ex. : «A la place des élèves, j'aurai fait pareil! » }\end{array}$ \\
\hline \multirow{5}{*}{ 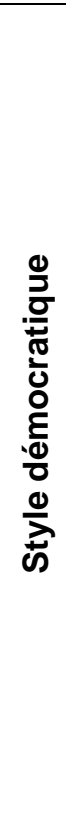 } & Clarification (clar) & $\begin{array}{l}\text { Consiste à faire préciser au stagiaire ses idées en lui posant } \\
\text { une ou plusieurs questions. } \\
\text { Ex. : «Peux-tu préciser ce qui s'est passé à ce moment-là ? » }\end{array}$ \\
\hline & Discussion (disc) & $\begin{array}{l}\text { Consiste à prendre en considération l'opinion du stagiaire en } \\
\text { lui demandant ce qu'il pense d'un sujet précis. } \\
\text { Ex. : « Ne penses-tu pas que... » }\end{array}$ \\
\hline & Confrontation (conf) & $\begin{array}{l}\text { Consiste à échanger son point de vue ou ses perceptions } \\
\text { avec ceux du stagiaire dans le but de soulever d'éventuelles } \\
\text { contradictions. } \\
\text { Ex.: « Je ne sais ce que tu en penses, mais moi j'ai } \\
\text { l'impression que... »; « tu avais dit que ferait ceci, mais tu ne } \\
\text { l'as pas fait» }\end{array}$ \\
\hline & Démonstration (demo) & $\begin{array}{l}\text { Consiste à illustrer certaines situations précises à partir de sa } \\
\text { propre expérience personnelle d'enseignant. } \\
\text { Exp. : « Moi souvent, ce que je fais, c'est que... » }\end{array}$ \\
\hline & Conseil (cons) & $\begin{array}{l}\text { Consiste à donner son avis en suggérant sa propre manière } \\
\text { de faire ou en proposant sa solution au problème. } \\
\text { Ex. : «Tu pourrais par exemple faire ceci, peut-être faire } \\
\text { cela... » }\end{array}$ \\
\hline \multirow{3}{*}{ 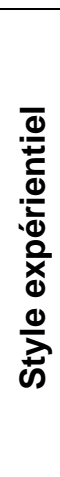 } & Exploration (expl) & $\begin{array}{l}\text { Consiste à stimuler le stagiaire à exprimer ce qu'il ressent en } \\
\text { l'invitant à se centrer sur ses propres préoccupations. } \\
\text { Ex.: « Je ne sais pas comment toi tu l'as ressenti ?» ; } \\
\text { «Quelles sont tes impressions?» }\end{array}$ \\
\hline & Soutien (sout) & $\begin{array}{l}\text { Consiste à se soucier de ce que vit le stagiaire tout en mettant } \\
\text { l'accent sur les points positifs de son intervention. } \\
\text { Ex. : « Je comprends que c'est pas facile... » « C'est légitime } \\
\text { de...» }\end{array}$ \\
\hline & Permissivité (perm) & $\begin{array}{l}\text { Consiste à laisser le stagiaire découvrir et expérimenter par } \\
\text { lui-même sa propre façon d'intervenir. } \\
\text { Ex. : « C'est à toi de faire comme tu le sens... » }\end{array}$ \\
\hline
\end{tabular}




\begin{tabular}{|c|c|}
\hline \multicolumn{2}{|c|}{ Annexe 2 - Définition des 10 catégories « objets de supervision » } \\
\hline Objectifs (obj) & $\begin{array}{l}\text { Concerne les buts, les intentions ou les attentes déterminés par le } \\
\text { stagiaire avant ou pendant la séance. Ils peuvent porter soit sur } \\
\text { l'amélioration d'un ou plusieurs aspect(s) de son propre } \\
\text { enseignement (obj. de formation); soit sur une amélioration de } \\
\text { l'apprentissage des élèves (obj. d'appr.). Ils peuvent concerner une } \\
\text { tâche, la séance, un cycle... }\end{array}$ \\
\hline Préparation (prepa) & $\begin{array}{l}\text { Concerne l'ensemble des tâches organisées et prévues par le } \\
\text { stagiaire avant la séance, à partir de processus cognitifs visant } \\
\text { l'anticipation des actions, sous la forme de scénarios. Les tâches y } \\
\text { sont décrites ainsi qu'une organisation préventive y est associée. }\end{array}$ \\
\hline Contenus (cont) & $\begin{array}{l}\text { Concerne les objets de savoirs spécifiques à chaque discipline } \\
\text { mobilisés par le stagiaire en cours de séance. Ces savoirs sont } \\
\text { relatifs au programme officiel, en rapport aux quatre axes de } \\
\text { compétence à développer chez les élèves (santé, sportif, sécurité et } \\
\text { expression). }\end{array}$ \\
\hline $\begin{array}{l}\text { Techniques de } \\
\text { communication } \\
\text { (tcom) }\end{array}$ & $\begin{array}{l}\text { Concerne l'utilisation, par le stagiaire, des habiletés } \\
\text { d'enseignement apprises en formation initiale, à savoir : la } \\
\text { démonstration, la communication verbale, la communication non } \\
\text { verbale, les rassemblements (début et fin de séance), les feedbacks } \\
\text { et l'observation. }\end{array}$ \\
\hline Orga & $\begin{array}{l}\text { Concerne les tâches de gestion de classe, comprenant la gestion du } \\
\text { groupe et du matériel dans l'espace-temps de la classe. Ils } \\
\text { concernent également les tâches habituelles de gestion des } \\
\text { transitions ainsi que de toutes les activités connexes non } \\
\text { directement reliées à l'apprentissage (vestiaire, constitution des } \\
\text { groupes, prise de présence). }\end{array}$ \\
\hline $\begin{array}{l}\text { Ordre et di } \\
\text { (ordr) }\end{array}$ & $\begin{array}{l}\text { Concerne l'établissement, au maintien et au contrôle des règles à } \\
\text { respecter par les élèves, condition indispensable pour assurer des } \\
\text { relations propices à l'apprentissage. II s'agit tant des règles de } \\
\text { sécurité, institutionnelles, de jeux ou encore d'apprentissage. }\end{array}$ \\
\hline $\begin{array}{l}\text { Relation avec les } \\
\text { élèves (rel) }\end{array}$ & $\begin{array}{l}\text { Concerne le climat relationnel (contrôlant ou soutenant l'autonomie) } \\
\text { mis en place par le stagiaire avec sa classe. II s'agit de l'attitude } \\
\text { positive, neutre ou négative de l'enseignant en interaction avec ses } \\
\text { élèves. Cette attitude est liée à la motivation du stagiaire } \\
\text { (enthousiasme, ennui, désintérêt...) }\end{array}$ \\
\hline Éthique (eth) & $\begin{array}{l}\text { Concerne les valeurs morales transmises par le stagiaire, pour } \\
\text { développer leur potentiel humain des élèves (éducation à la } \\
\text { citoyenneté). }\end{array}$ \\
\hline Réflexivité (reflex) & $\begin{array}{l}\text { Concerne la capacité à analyser, à expliciter et à réfléchir sur sa } \\
\text { pratique à partir de sa propre expérience. Cette capacité passe par } \\
\text { une mise à distance du vécu, et par une remise en question } \\
\text { éventuelle. Le but de cette démarche est d'en comprendre le sens, } \\
\text { et d'ajuster sa pratique si nécessaire. }\end{array}$ \\
\hline $\begin{array}{l}\text { Vécu personnel } \\
\text { (vecu) }\end{array}$ & $\begin{array}{l}\text { Concerne tout ce que vit intérieurement le stagiaire, autrement dit } \\
\text { son ressenti subjectif, comprenant ses préoccupations, ses } \\
\text { perceptions, ses sensations, ses sentiments et ses émotions. }\end{array}$ \\
\hline
\end{tabular}

